Viscous linear stability analysis of rectangular duct and cavity flows

Theofilis, V. and Duck, P. and Owen, J.

2004

MIMS EPrint: 2006.251

Manchester Institute for Mathematical Sciences

School of Mathematics

The University of Manchester

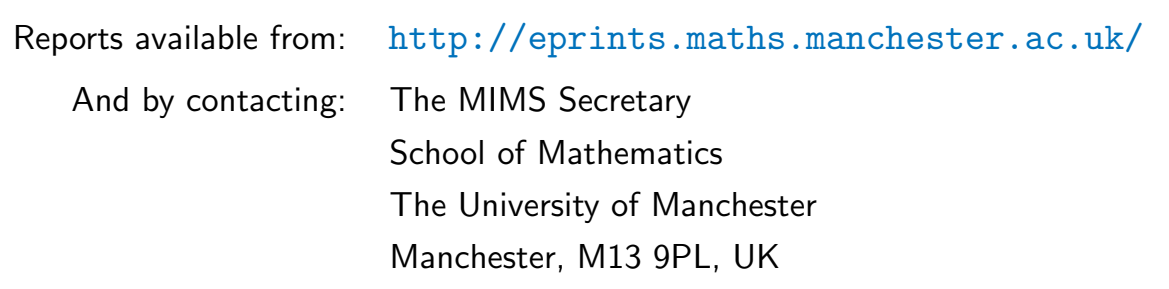

ISSN 1749-9097 


\title{
Viscous linear stability analysis of rectangular duct and cavity flows
}

\author{
By V. THEOFILIS ${ }^{1}$, P. W. DUCK ${ }^{2}$ AND J. OWEN ${ }^{2}$ \\ ${ }^{1}$ Escuela Técnica Superior Ingenieros Aeronáuticos, Universidad Politécnica de Madrid, \\ Pza. Cardenal Cisneros 3, E-28040 Madrid, Spain \\ ${ }^{2}$ Department of Mathematics, University of Manchester, Manchester M13 9PL, UK
}

(Received 2 May 2001 and in revised form 17 December 2003)

The viscous linear stability of four classes of incompressible flows inside rectangular containers is studied numerically. In the first class the instability of flow through a rectangular duct, driven by a constant pressure gradient along the axis of the duct (essentially a two-dimensional counterpart to plane Poiseuille flow - PPF), is addressed. The other classes of flow examined are generated by tangential motion of one wall, in one case in the axial direction of the duct, in another perpendicular to this direction, corresponding respectively to the two-dimensional counterpart to plane Couette flow (PCF) and the classic lid-driven cavity (LDC) flow, and in the fourth case a combination of both the previous tangential wall motions. The partial-derivative eigenvalue problem which in each case governs the temporal development of global three-dimensional small-amplitude disturbances is solved numerically. The results of Tatsumi \& Yoshimura (1990) for pressure-gradient-driven flow in a rectangular duct have been confirmed; the relationship between the eigenvalue spectrum of PPF and that of the rectangular duct has been investigated. Despite extensive numerical experimentation no unstable modes have been found in the wall-bounded Couette flow, this configuration found here to be more stable than its one-dimensional limit. In the square LDC flow results obtained are in line with the predictions of Ding \& Kawahara (1998b), Theofilis (2000) and Albensoeder et al. (2001b) as far as one travelling unstable mode is concerned. However, in line with the predictions of the latter two works and contrary to all previously published results it is found that this mode is the third in significance from an instability analysis point of view. In a parameter range unexplored by Ding \& Kawahara (1998b) and all prior investigations two additional eigenmodes exist, which are both more unstable than the mode that these authors discovered. The first of the new modes is stationary (and would consequently be impossible to detect using power-series analysis of experimental data), whilst the second is travelling, and has a critical Reynolds number and frequency well inside the experimentally observed bracket. The effect of variable aspect ratio $A \in[0.5,4]$ of the cavity on the most unstable eigenmodes is also considered, and it is found that an increase in aspect ratio results in general destabilization of the flow. Finally, a combination of wall-bounded Couette and LDC flow, generated in a square duct by lid motion at an angle $\phi \in(0, \pi / 2)$ with the homogeneous duct direction, is shown to be linearly unstable above a Reynolds number $R e=800$ (based on the lid velocity and the duct length/height) at all $\phi$ parameter values examined. The excellent agreement with experiment in LDC flow and the alleviation of the erroneous prediction of stability of wall-bounded Couette flow is thus attributed to the presence of in-plane basic flow velocity components. 


\section{Introduction}

Linear stability theory is concerned with the evolution of small-amplitude disturbances superimposed upon a basic state. In the case of steady basic flows, separability of time from the three spatial coordinates in the disturbance equations suggests that an eigenmode Ansatz may be introduced to describe the time-evolution of disturbances, their amplitude functions being dependent on the three spatial coordinates in general. However, numerical solution of the fully three-dimensional (global) linear eigenvalue problems is still impractical using currently available hardware and algorithms for all but the smallest Reynolds number values (e.g. E. Leriche 2003, personal communication).

In order to perform a temporal linear stability analysis of flows varying in more than one dimension a number of simplifications have been employed in the past, often in a heuristic fashion, the most radical of which considers a basic state to depend on one spatial coordinate alone, the other two spatial directions being taken as homogeneous, thereby permitting the use of normal-mode analysis in the latter two directions. This simplified approach has been widely employed in the last century to study (for example) the instability of boundary layers where the basic flow velocity component in the wall-normal direction is usually neglected, together with boundary-layer growth effects, assumptions commonly known as the parallel-flow approximation.

The predictions of classical linear stability theory have met with mixed success. The most notable verification of the theory was the experimental identification by Schubauer \& Skramstad (1947) of the instability waves on a flat-plate boundary layer postulated by Tollmien (1929); the most notable failure of the theory is its prediction of stability of Hagen-Poiseuille flow (HPF) in a pipe at all Reynolds numbers, although instability and transition to turbulence in this flow is known at least since the celebrated experiments of Reynolds (1883); the case of (one-dimensional) plane Couette flow (PCF) is another glaring anomaly. These discrepancies are all the more alarming, given that the basic flows in these two latter states are both truly parallel.

Between the two extremes of basic flows dependent upon one coordinate direction and flows dependent upon all three coordinates, one may consider steady or time-periodic basic flows dependent upon two spatial directions and impose threedimensional disturbances periodic in the third, homogeneous, spatial direction. The non-parallel (global) linear instability analysis that forms the basis of the present paper constitutes the natural extension of the classic linear stability theory eigenvalue problem (Tollmien 1929) in that, in place of the system of the ordinary-differential Orr-Sommerfeld and Squire equations which result from the one-dimensional basic flow assumption, a system of partial differential equations is considered. The link with classical analyses may (in some cases) be achieved in a self-consistent manner as a limiting case of solutions to the partial-derivative eigenvalue problem, as shown in this paper and in the recent review of Theofilis (2003), where an extensive discussion of the present analysis methodology, also known as BiGlobal linear theory (Theofilis et al. 2003; Jacquin et al. 2003; Seifert et al. 2004), may be found.

Results obtained using global instability analysis are slowly emerging in many areas of fluid mechanics, following the pace of hardware and algorithmic developments. The first studies of the partial derivative eigenvalue problem arising in fluid dynamics focused on the inviscid limit of certain problems, notably by Pierrehumbert (1986), who reported the discovery of short-wavelength instability in inviscid vortex flow. Henningson (1987) studied the stability of inviscid shear flows with mean flow spanwise variation while Hall \& Horseman (1991) addressed the secondary instability of Görtler vortices, potentially appearing in regions of surface or streamline curvature 
on aircraft components. All three studies involved the solution of what may be regarded as the two-dimensional analogue of the Rayleigh problem, since the analysis was based upon inviscid disturbance equations. Recently Otto \& Denier (1999) have presented an efficient method for solution of these types of equations.

Early work which has included the effect of viscosity in the global stability analysis is that of Lee, Schultz \& Boyd (1989) who addressed low-Rayleigh-number heat transfer in a rectangular container and Tatsumi \& Yoshimura (1990) who were the first to solve the generalized Orr-Sommerfeld and Squire system for the flow driven by a constant pressure gradient inside a rectangular duct. Morzynski $\&$ Thiele (1991) and Barkley \& Henderson (1996) addressed the instability of laminar flow behind a circular cylinder, while Barkley, Gomes \& Henderson (2002) solved the linear instability of laminar flow at the backward facing step. Lin \& Malik (1996) applied global linear instability theory to study the swept (Hiemenz) attachmentline boundary-layer flow, a problem also solved by Theofilis et al. (2003), who demonstrated that the three-dimensional global linear eigenmodes of this flow may be modelled and recovered as solutions of a sequence of one-dimensional eigenvalue problems of the Orr-Sommerfeld class. Ehrenstein (1996) solved the global eigenvalue problem in a channel one wall of which was modelling a riblet geometry and showed that the primary instability of this channel flow was enhanced when compared with the classic plane Poiseuille flow (PPF). Wintergerste \& Kleiser (2000) studied the stability of nonlinearly generated crossflow vortices during the late transitional stages of boundary-layer flow, while Härtel \& Meiburg (1999) have applied global linear theory to explain their observations in direct numerical simulations (DNS) of avalanches.

In this paper we are concerned with flows inside rectangular containers. The first class of flow considered is that in a rectangular duct of cross-sectional aspect ratio $A$, driven by a constant pressure gradient along the axial (unbounded) direction; the large-aspect-ratio $(A \rightarrow \infty)$ limit of this configuration is PPF. The second class of flow considered is wall-bounded Couette flow in a rectangular enclosure, one wall of which is sliding in the axial direction of the duct, and so the limit of large aspect ratio relates this basic flow with the classic (one-dimensional) PCF profile. If on the other hand the direction of motion of the sliding wall is perpendicular to the axial direction of the rectangular domain, a lid-driven cavity (LDC) flow is obtained; in the latter two flows the pressure gradient along the unbounded duct direction is taken to be zero. Finally, the two extremes of wall-bounded Couette and lid-driven cavity flows may be reconciled by considering a lid motion along a direction between the respective extreme values. In general there is no rational approximation which reduces the instability problem in any of these four flows to a set of ordinary-differential equations.

The stability of pressure-gradient-driven flow through a rectangular duct has attracted much interest in recent times. Kao \& Park (1970) performed controlled transition experiments in an $A=8$ flow and concluded that the flow is unstable above a critical Reynolds number $R e_{\text {crit }} \approx 2600$ and wavenumber $\beta_{\text {crit }} \approx 1.5$. These authors also applied classical linear theory, based on the solution of the Orr-Sommerfeld equation, in an effort to relate the measured disturbance amplitude functions to the eigenfunctions of PPF. They obtained fair agreement as far as the eigenfunctions were concerned but not with respect to the critical parameter values. Tatsumi \& Yoshimura (1990) in their influential work addressed the problem of viscous linear instability of the rectangular duct flow by applying the appropriate partial derivative eigenvalue problem analysis. They concluded that the lateral wall has a stabilizing effect on PPF, 
and showed that a decrease of the aspect ratio leads to an increase of the critical Reynolds number from the overprediction $R e_{\text {crit,PPF }}=5772.22$ that linear theory yields in the limit $A \rightarrow \infty$ (Orszag 1971); they postulated that $R e_{\text {crit }} \rightarrow \infty$ at $A=1$, thus adding another paradox (within an eigenvalue-problem stability analysis) of linear stability of flow in the square duct alongside that of HPF in a pipe. Tatsumi \& Yoshimura (1990) went on to attribute the discrepancy between their instability results and experiment to an unquantified nonlinear instability mechanism. As a matter of fact, subcritical instability is known to be responsible for transition to turbulence in the related problem of PPF, as Herbert $(1974,1977)$ has demonstrated. DNS work (Kleiser 1982; Orszag \& Patera 1983; Zang \& Hussaini 1995) has elaborated on this mechanism and shed additional light on the specific nonlinear mode interactions. These theoretical results have been supported by the careful experiments of Nishioka, Iida \& Ichikawa (1975) and Nishioka \& Asai (1985). An analogous theory for subcritical instability of rectangular duct flow does not exist at present.

Kerswell \& Davey (1996) discussed the linear instability of flow in a pipe with elliptical cross-section. They found that the constant-pressure-gradient-driven flow in a relatively small-aspect-ratio geometry, $A \leqslant 5$, is linearly stable and were able to identify the instability of the spanwise modulated analogue of the most unstable PPF eigenmode above $A \approx 10.5$. Their large-aspect-ratio results were in line with the predictions of Hocking $(1977,1978)$ who proposed that the spanwise boundary curvature introduced by the sidewalls in a rectangular duct has a stabilizing effect on the flow, when compared with its infinite-aspect-ratio PPF counterpart, and put forward the relationship

$$
R e_{\text {crit }}=R e_{\text {crit, } \mathrm{PPF}}+\frac{6844}{A^{2}}
$$

to relate the critical Reynolds number in a duct of aspect ratio $A$ to that of PPF. Though qualitatively correct, the quantitative comparison with the subsequent numerical results of Tatsumi \& Yoshimura (1990) did not confirm the above asymptotic relationship, pointing to the need for numerical approaches to provide accurate predictions. For small-aspect-ratio elliptic pipe and rectangular duct flows, as well as their idealized analogues, HPF and PPF, this discussion is largely academic since linear theory has failed to match experimental observation. Using experiment and computation, Tumin (2000) has recently discussed the onset of turbulence in circular pipe flow from a nonlinear instability point of view, and singled out the occurrence of hairpin vortices as a universal characteristic of pipe flow transition, which may encompass both secondary instability (Herbert 1977) and transient growth scenarios (Trefethen et al. 1993; Schmid \& Henningson 2001).

No work is known with respect to the stability of Couette flow in the presence of lateral walls. It is worth emphasizing that the simplicity of the basic flow in the one-dimensional case has prompted intense investigation of the linear instability of PCF, but has led to what until recently was considered a paradox, namely linear stability theory failing to locate any unstable modes, first in a multitude of numerical approaches and secondly in the analytical work of Romanov (1973). Long ago Rayleigh (1914) attributed transition to turbulence in PCF to finite-amplitude disturbances. Nonlinearity and non-normality of the linear operator in shear flows are the central themes in the experimental works of Daviaud, Hegseth \& Bergé (1992), Tillmark \& Alfredsson (1992) and Bottin et al. (1998), the combined theoretical and numerical work of Eckhardt, Marzinzik \& Schmiegel (1998), Faisst \& Eckhardt (2000) as well as the theoretical works of Waleffe (1995), Boberg \& Brosa (1988) and 
Trefethen et al. (1993). Nonlinear analysis in the works of Nagata (1990) and Clever \& Busse (1997) delivered the first three-dimensional structures in PCF. Discussion of both issues is beyond the scope of the present paper, where we focus instead on studying the effect of lateral walls on the stability of PCF.

The problem of calculating the steady flow inside a square cavity, driven by the sliding of the cavity lid, has interested fluid dynamicists and numerical analysts alike for over 30 years. The intricacies of the basic-flow problem were found originally by Burggraf (1966); the flow is rich in fluid mechanics, even at modest Reynolds numbers, comprising a core vortex and vortices located in the corners of the container, created as a result of flow separations. Indeed local analysis, based on that of Moffatt (1964) indicates that infinite solution resolution would lead to an infinite number of progressively weaker counter-rotating eddies in these lower-corner regions. The singular solution behaviour at the top corners of the container further complicates the flow and numerical analysis - the simplicity of the geometry is not matched by the flow solution per se. At Reynolds numbers above just a few hundred (the régime studied by Burggraf 1966), more sophisticated numerical analysis and computational procedures are required, and it is this that has led to this problem becoming a benchmark for workers in the field of the numerical solution of elliptic partial-differential equations. Early numerical solutions focused on the steady equations of motion and accuracy was limited by the then available hardware capabilities. Unsteady approaches to the problem yield a yet further twist to the overall picture. The work of Goodrich, Gustafson \& Halasi (1990) and Shen (1991) indicates that the flow converges to a (two-dimensional) steady state for Reynolds numbers up to about $10^{4}$. The former work suggests that for Reynolds numbers in the range $10000<R e<10500$ the flow becomes temporally periodic with a Hopf bifurcation, whilst Shen (1991) shows the flow to become quasi-periodic in the range $15000<R e<15500$ in the regularized case, with a non-uniform sliding of the lid (in order to avoid difficulties with flow singularities at the top corners of the cavity).

Pan \& Acrivos (1967) conducted a series of experiments with relatively deep (i.e. small-aspect-ratio) cavities, and obtained some quite encouraging agreement with the numerical results of Burggraf (1966). These experiments were conducted in the range $20<R e<4000$ and indicated that an increase in the (cavity-width-based) Reynolds number causes the centre of the core vortex to move to the centre of the cavity, and the size of the so-called downstream eddy (that is the eddy at the lower righthand-side corner, assuming the lid to move from left to right) increases in size up to a Reynolds number of 500, and thereafter diminishes in scale. Koseff \& Street (1984) and Freitas et al. (1985) conducted visualization studies on flows inside square cavities of spanwise aspect ratio $3: 1$, in the range $1000<R e<10000$. Up to $R e=3200$ the flow was observed to be two-dimensional, but beyond this value the flow became discernibly three-dimensional, with the formation of counter-rotating (Taylor-Görtlerlike) vortices near the downstream eddy with an axis parallel to that of the moving lid. In the flow regime $R e>5000$ it was observed that the flow became turbulent. Aidun, Triantafillopoulos \& Benson (1991) refined the observations of previous investigations by conducting experiments on the flow inside a lid-driven cavity (with depth- and span-to-width aspect ratios of $1: 1$ and $3: 1$ respectively), and concluded that for Reynolds numbers below 500 the flow was two-dimensional, between $825<R e<925$ the flow underwent a transition to a three-dimensional, small-amplitude, time-periodic state. For $1000<R e<1300$ travelling waves were seen to appear, with a much more complicated flow structure. For Reynolds numbers in excess of 1900 irregularly spaced mushroom-like structures were observed. These authors also observed the existence 
of multiple steady states $\dagger$. Later, Benson \& Aidun (1992) by using a power-spectral analysis of their experimental results, estimated the dimensionless neutral frequency for instability to be 0.1112 .

Three-dimensional DNS of the problem has been undertaken by Kim \& Moin (1985) who employed periodic boundary conditions in the spanwise direction and indicated the occurrence of two pairs of Taylor-Görtler vortices when $R e>900$ (although just one spanwise wavenumber was considered, and it is unclear if the vortices were steady). Ku, Hirsh \& Taylor (1987) also performed a three-dimensional simulation, but within a cubic cavity (with no slip replacing periodicity in the spanwise direction), and found the effect of the endwalls was to suppress the Taylor-Görtler vortices. More recent work using different strategies for the numerical integration of the incompressible Navier-Stokes and continuity equations in a cubic cavity (e.g. Guj \& Stella 1993; Tang, Cheng \& Tsang 1995) has produced results which are essentially in agreement with those of Ku, Hirsh \& Taylor (1987) at $R e=1000$ as far as the streamwise and normal velocity profiles along the centre axes on the spanwise midplane are concerned. However, while Tang et al. (1995) have demonstrated evidence of Taylor-Görtler vortices, Guj \& Stella (1993) have failed to report them.

When seeking an explanation of these apparently contradictory results of two- and three-dimensional cavities one may attempt to use global linear instability analysis to investigate whether the different phenomena observed might be attributed to the different growth/damping rates of disturbances pertaining to different wavenumbers of three-dimensional global eigenmodes. Calculations of this type are extremely challenging; the difficulties in computing the basic state have already been alluded to, and it is well known that instability analyses are extremely sensitive to the accuracy of the prescribed basic state (which is two-dimensional). We are led, therefore, to consider the solution of partial-eigenvalue problems, which are very demanding in computational resources; again, recent developments in algorithms and computational hardware have enabled significant progress to be made in this area.

The first attempt at studying the stability of lid-driven cavity flows appears to be that by Poliashenko \& Aidun (1995) who analysed the stability of the flow to twodimensional global modes (i.e. those pertaining to zero spanwise wavenumber) and found a subcritical Hopf bifurcation at a Reynolds number of about 7000 for cavity aspect ratios around unity. However the aforementioned experimental observations all point to the fact that it is three-dimensional disturbances which are the first to become unstable (clearly, in this context, the use of Squires' theorem is entirely inappropriate). Ramanan \& Homsy (1994) used a high-order finite-difference scheme on the problem, and determined a loss of stability in the square cavity case at a critical Reynolds number of 594, the instability mechanism being of zero frequency, with nondimensional wavenumber (in the third/spanwise direction) of $\beta=2.12$. Three papers by Ding \& Kawahara $(1998 a, b, 1999)$ considered the problem using a finite-element methodology. In the first paper the authors indicated the existence of Taylor-Görtlerlike vortices in the cavity; in this case a critical Reynolds number of 1025 was predicted, together with critical non-dimensional spanwise wavenumber and frequency values of $\left(\beta_{\text {crit }}, f_{\text {crit }}\right) \approx(7.6,0.080)$. The second paper by the same authors predicted a critical Reynolds number of 925 , together with critical parameters $(7.4,0.079)$, while the third paper refined the critical Reynolds number estimate to $R e \approx 920.3$ and

$\dagger$ The existence of multiple steady states in the related problem of flow in a cavity driven by two opposite walls moving along the same or in the opposite direction has recently been documented by Albensoeder, Kuhlmann \& Rath (2001a) 
confirmed the critical wavenumber and frequencies of Ding \& Kawahara (1998b). The assertion was also put forward by these authors that the instability observed is an absolute instability in the sense of Huerre \& Monkewitz (1990). It might be argued that this classification is confusing since in lid-driven cavity flow, where flow develops inside a closed container, the concept of upstream propagation of a localized disturbance is not appropriate since the flow is not unidirectional. In any event, one might consider the agreement between the frequencies of the least-stable mode calculated theoretically in the works of Ding \& Kawahara $(1998 a, b, 1999)$ and that observed experimentally (Benson \& Aidun 1992) as fair. However, the discrepancy between this frequency and that calculated by Ramanan \& Homsy (1994), as well as the discrepancy in the critical Reynolds numbers presented in the previous investigations is unsatisfactorily wide. Aside from the inconsistency in the results of previous work, open questions also leave some concern regarding the large discrepancies with the numerical results in the DNS of Kim \& Moin (1985), who present in their figure 7 the most unstable wavenumber $\beta=2 \pi / L_{z} \approx 2 \pi / 0.533=11.79$. We note that all four instability analyses discussed agree on the existence of unstable modes in the region $\beta \in[0,10]$ but no information is available for higher wavenumbers. Theofilis (2000) and Albensoeder, Kuhlmann \& Rath (2001b) have revisited the problem of square LDC instability, while the latter investigators have also reported critical conditions in the case of rectangular cavity flows. Clearly, there is some variance in the previously published data on the LDC problem, and one of the objectives of the present paper is to produce rather more definitive results.

Finally, a combined wall-bounded-Couette/LCD flow is examined in an attempt to reconcile the instability analysis results in the two extremes of moving-lid direction. In this case an additional parameter is introduced in the problem, namely the angle $\phi$ between the lid motion and the axial direction of the duct. Besides providing the link between the PCF and LDC flows, the combined wall-bounded-Couette/LDC flow serves as a model of three-dimensional flow in open cavities at high speeds, where experimental and numerical work has related hypersonic flow in an open cavity to incompressible LDC flow (Jackson, Hillier \& Soltani 2001). Despite its relative complexity, such a model offers substantial simplifications in instability investigations of open cavities (Rowley, Colonius \& Basu 2002) and represents the first effort employing non-parallel global instability analysis techniques to a cavity flow comprising a three-component velocity vector. Three representative values of the angle $\phi$, alongside Reynolds number values either side of the linear critical limits pertaining to each eigenmode, and a full scan of the wavenumber space have been considered in this fourth class of flows.

The outline of this paper is as follows. In $\S 2$ details of the steady laminar twodimensional basic flows which are subsequently analysed are presented. In $\S 3$ the theoretical foundation of the global non-parallel linear instability analysis is discussed, alongside algorithmic considerations regarding the challenging numerical solution of the partial derivative eigenvalue problem. Stability results for all four classes of flow are presented in $\S 4$ and conclusions are drawn in $\S 5$.

\section{Two-dimensional basic flows}

Consider a rectangular domain - a schematic representation is shown in figure 1 . If all walls are stationary a steady laminar flow may be set up inside the duct by imposing a constant pressure gradient along the homogeneous $(z)$ spatial direction. On the other hand, in the absence of a pressure gradient, distinct steady laminar 


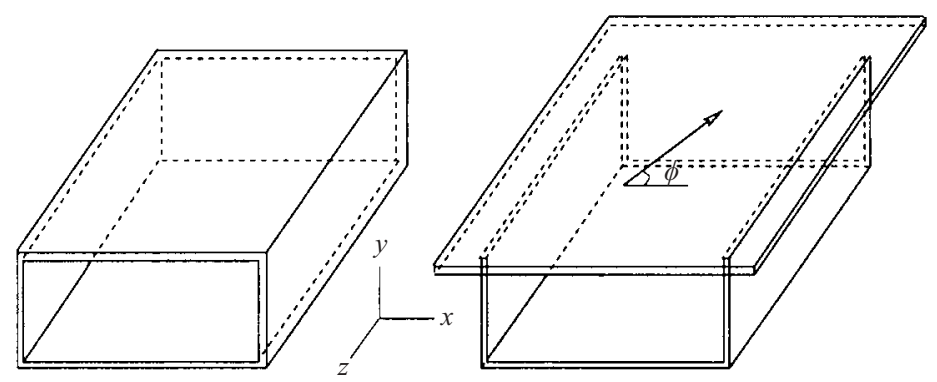

FIGURE 1. Rectangular duct flow configurations addressed.

flow patterns result from uniform motion of the top wall at a constant velocity in a direction at an angle $0 \leqslant \phi \leqslant \pi / 2$ with the $x$-axis. The two limiting cases, $\phi=\pi / 2$ and $\phi=0$, give rise to a wall-bounded two-dimensional Couette (2DC) flow and lid-driven cavity (LDC) flow (Burggraf 1966), respectively. The non-parallel linear instability analysis of the steady laminar flows in the rectangular duct and the two latter flows is the subject of the present investigations. The instability analysis of the steady laminar flows resulting from $0<\phi<\pi / 2$ corresponds to the most general family of flows comprising three velocity components and presents an order-of-magnitude greater challenge in terms of computing requirements compared with the computing effort necessary for the previous three classes of flows. Basic flows have been obtained in this case for a square domain and a small number of parameters $(R e, \phi)$, sufficient to elucidate the link between the 2DC and LCD flows.

\subsection{Pressure-gradient-driven flow through a rectangular duct}

Consider the rectangular duct defined in the domain $\Omega=\{x \in[-A, A]\} \times\{y \in$ $[-1,1]\}$, where $A$ is the aspect ratio; all length scales here have been nondimensionalized with respect to the duct semi-depth. A constant pressure gradient in the unbounded $(z)$ direction drives a steady laminar flow which is independent of $z$ and possesses a velocity vector $(0,0, \bar{w})^{\mathrm{T}}$ with a single velocity component $\bar{w}(x, y)$ along the $z$ spatial direction, which satisfies the Poisson equation

$$
\nabla_{2 d}^{2} \bar{w}(x, y)=-\partial \bar{p} / \partial z=c,
$$

where $\nabla_{2 d}^{2}=\partial^{2} / \partial x^{2}+\partial^{2} / \partial y^{2}$. The boundary conditions are

$$
\bar{w}(x, y=-1)=\bar{w}(y, x=-A)=\bar{w}(x, y=1)=\bar{w}(x=A, y)=0 .
$$

Taking $c=-2$ in (2.1) and scaling the result with the value of $\bar{w}$ at the midpoint of the integration domain, the Poisson problem may be solved in series form (Rosenhead 1963)

$$
\bar{w}(x, y)=1-y^{2}-4\left(\frac{2}{\pi}\right)^{3} \sum_{n=0}^{\infty} \frac{(-1)^{n}}{(2 n+1)^{3}} \frac{\cosh [(2 n+1) \pi x / 2] \cos [(2 n+1) \pi y / 2]}{\cosh [(2 n+1) \pi A / 2]} .
$$

The plane Poiseuille basic flow result, $\bar{w}(y)=1-y^{2}$, is retrieved from this expression in the limit $A \rightarrow \infty$. However we chose to undertake a fully numerical solution for the basic flow, using spectral collocation for the discretization and numerical solution of (2.1)-(2.2) in the rectangular domain in question, with analytical mappings used to transfer information from the latter on to the canonical Legendre Gauss-Lobatto grids. An acceptable approximation to the analytical result (2.3) is obtained on just a $8^{2}$ grid on which the centrepoint velocity differs from the analytical solution by less 


\begin{tabular}{|c|c|c|c|c|c|c|c|}
\hline \multicolumn{2}{|c|}{$A=1$} & \multicolumn{6}{|c|}{$A=2$} \\
\hline$\left(N_{x} \times N_{y}\right)$ & $Q_{\mathrm{nd}}$ & $\left(N_{x} \times N_{y}\right)$ & $Q_{\mathrm{nd}}$ & $\left(N_{x} \times N_{y}\right)$ & $Q_{\text {nd }}$ & $\left(N_{x} \times N_{y}\right)$ & $Q_{\text {nd }}$ \\
\hline $\begin{array}{c}8 \times 8 \\
16 \times 16 \\
24 \times 24 \\
32 \times 32\end{array}$ & $\begin{array}{l}0.25031 \\
0.25003 \\
0.25001 \\
0.25000\end{array}$ & $\begin{array}{c}8 \times 8 \\
16 \times 16 \\
24 \times 24 \\
32 \times 32\end{array}$ & $\begin{array}{l}0.36458 \\
0.36475 \\
0.36478 \\
0.36479\end{array}$ & $\begin{array}{l}16 \times 8 \\
32 \times 16 \\
48 \times 24 \\
64 \times 32\end{array}$ & $\begin{array}{l}0.36497 \\
0.36482 \\
0.36480 \\
0.36479\end{array}$ & $\begin{array}{l}16 \times 8 \\
16 \times 32 \\
24 \times 48 \\
32 \times 64\end{array}$ & $\begin{array}{l}0.36414 \\
0.36470 \\
0.36477 \\
0.36478\end{array}$ \\
\hline \multicolumn{4}{|c|}{ analytical $Q_{\mathrm{nd}}=0.25$} & \multicolumn{4}{|c|}{ analytical $Q_{\mathrm{nd}}=0.36479$} \\
\hline \multicolumn{4}{|c|}{$A=3$} & \multicolumn{4}{|c|}{$A=4$} \\
\hline$\left(N_{x} \times N_{y}\right)$ & $Q_{\mathrm{nd}}$ & $\left(N_{x} \times N_{y}\right)$ & $Q_{\text {nd }}$ & $\left(N_{x} \times N_{y}\right)$ & $Q_{\mathrm{nd}}$ & $\left(N_{x} \times N_{y}\right)$ & $Q_{\text {nd }}$ \\
\hline $\begin{array}{c}8 \times 8 \\
16 \times 16 \\
24 \times 24 \\
32 \times 32\end{array}$ & $\begin{array}{l}0.40886 \\
0.40945 \\
0.40953 \\
0.40955\end{array}$ & $\begin{array}{l}24 \times 8 \\
48 \times 16 \\
72 \times 24 \\
96 \times 32\end{array}$ & $\begin{array}{l}0.40969 \\
0.40957 \\
0.40956 \\
0.40955\end{array}$ & $\begin{array}{l}16 \times 16 \\
24 \times 24 \\
32 \times 32 \\
36 \times 36\end{array}$ & $\begin{array}{l}0.43199 \\
0.43212 \\
0.43214 \\
0.43215\end{array}$ & $\begin{array}{r}64 \times 16 \\
80 \times 20 \\
96 \times 24 \\
112 \times 28\end{array}$ & $\begin{array}{l}0.43217 \\
0.43216 \\
0.43216 \\
0.43215\end{array}$ \\
\hline \multicolumn{4}{|c|}{ analytical $Q_{\mathrm{nd}}=0.40956$} & \multicolumn{4}{|c|}{ analytical $Q_{\mathrm{nd}}=0.43216$} \\
\hline
\end{tabular}

than 1 part in $10^{8}$. This agreement is essential since, as already stated, the quality of the basic flow conditions that of the eigenvalue problem results.

\subsection{Two-dimensional Couette $(2 D C)$ flow}

In this problem we consider, as before, the rectangular domain $\Omega$ and take the basic flow again to have a single component and so the velocity vector can be written $(0,0, \bar{w}(x, y))^{\mathrm{T}}$. In this case the flow is driven by a uniform lid motion along the $z$-direction. The one velocity component satisfies (2.1) with $c=0$, subject to the boundary conditions

$$
\bar{w}(x=A, y)=\bar{w}(x, y=-1)=\bar{w}(x=-A, y)=0, \quad \bar{w}(x, y=1)=1 .
$$

It is straightforward to derive an analytical solution for $\bar{w}(x, y)$,

$$
\bar{w}(x, y)=\sum_{n=0}^{\infty} \frac{4(-1)^{n}}{(2 n+1) \pi} \frac{\sinh [(2 n+1) \pi(y+A) / 2]}{\sinh [(2 n+1) \pi A]} \cos [(2 n+1) \pi x / 2] .
$$

Note the corner singularities in (2.5) which will result in a deterioration of the exponential convergence of the spectral method for the numerical solution of (2.1) subject to (2.4) but not its ability to converge as the resolution increases. In table 1 we monitor the numerical solution of (2.1) subject to (2.4) at four aspect ratios, $A=1,2,3$ and 4 . We present results for the non-dimensional volume flux,

$$
4 A Q_{\mathrm{nd}}=\int_{y=-1}^{1} \int_{x=-A}^{A} \bar{w}(x, y) \mathrm{d} x \mathrm{~d} y=32 \frac{A^{2}}{\pi^{3}} \sum_{n=0}^{\infty} \frac{[\cosh (2 n+1) \pi / A-1]}{(2 n+1)^{3} \sinh (2 n+1) \pi / A},
$$

since this quantity provides an integral measure of the flow quality and delivers a much better indication of the resolution requirements for the eigenvalue problem compared with monitoring the convergence of isolated values of the basic flow velocity.

\subsection{Lid-driven cavity $(L D C)$ flow}

The third class of flow we will be investigating is that in a two-dimensional rectangular container filled with incompressible fluid and driven by a lid which moves with 
constant velocity perpendicular to the duct axis. As noted previously, the earliest study of this basic flow was undertaken by Burggraf (1966). There is a review of some of the early work on the problem by Tuann \& Olson (1978); there are many discrepancies between the different formulations in this era, no doubt due to the poor resolution restricted by computational limitations at that time. Later work, aided by the development of both computer hardware and numerical techniques, led to a much more satisfactory state of affairs. Ghia, Ghia \& Shin (1982) used a multigrid approach to obtain solutions up to a Reynolds number of $10^{4}$, the regime which was also computed by Schreiber \& Keller (1983) using a direct solution procedure, based on Newton's method. Other work includes that of Shay (1981), Thompson \& Ferziger (1989), Bruneau \& Jouron (1990), Napolitano \& Catalano (1991), Gupta (1991), Nishida \& Satofuka (1992) and Guj \& Stella (1993). This list is not supposed to be comprehensive, but merely indicative of the interest that this problem has aroused over many years.

Here we take $x$ to be in the direction of the motion of the lid and $y$ to be along the normal to this direction. The basic flow is considered independent of the third (spanwise) direction $z$, an assumption which has been shown by Ku, Hirsh \& Taylor (1987) to lead to different results compared to a flow that develops in containers of finite spanwise extent at all Reynolds numbers $R e \geqslant 10^{3}$. The steady basic flow vector under these assumptions has two velocity components, $(\bar{u}(x, y), \bar{v}(x, y), 0)^{\mathrm{T}}$, and only numerical solutions to this steady laminar basic flow are known.

For the present two-dimensional problem it is advantageous from a numerical point of view to consider the vorticity-transport equation

$$
\frac{\partial \zeta}{\partial t}+\frac{1}{R e} \nabla_{2 d}^{2} \zeta-\left\{\frac{\partial \psi}{\partial y} \frac{\partial \zeta}{\partial x}-\frac{\partial \psi}{\partial x} \frac{\partial \zeta}{\partial y}\right\}=0,
$$

with

$$
\nabla_{2 d}^{2} \psi+\zeta=0
$$

relating the streamfunction $\psi$ and vorticity $\zeta$. The velocity components are obtained through $(\bar{u}, \bar{v})=(\partial \psi / \partial y,-\partial \psi / \partial x)$. The boundary conditions are $\bar{v}=0$ on all four walls $x=0, x=A, y=0$ and $y=1, \bar{u}=0$ on $x=0, x=A$ and $y=0$. The one inhomogeneous condition is $\bar{u}(x, y=1)=1$. Note that the domain here $(0 \leqslant x \leqslant A$, $0 \leqslant y \leqslant 1$ ) is slightly different from that used in the previous two configurations, but this is deliberately chosen to conform with the nomenclature employed in the past on these problems. The mismatch at the ends of the moving lid in this case is expected to result in singularities analogous to those encountered in the two-dimensional Couette flow discussed previously. Indeed, it is observed that the presence of singular boundary conditions degrades convergence from spectral to algebraic. A time-marching procedure was employed to generate the steady state; in the light of the work of Goodrich et al. (1990) and Shen (1991), such a technique is likely to be successful for Reynolds numbers below about $10^{4}$, well beyond our envelope of investigation. Algorithmic details for the computation of the twodimensional steady state may be found elsewhere (Theofilis 2003). Here we note that in the streamfunction/vorticity-transport formulation all boundary conditions can be imposed indirectly on the derivatives of the streamfunction, whilst no conditions are imposed directly on the vorticity. Upon applying the semi-implicit scheme of Spalart, Moser \& Rogers (1991) for the time-integration of (2.7) a set of Poisson problems for the streamfunction and vorticity must be solved. Direct inversion of the matrices, though possible, is not efficient and we have implemented a matrix-diagonalization 
technique. If $N_{x}$ and $N_{y}$ points are used to resolve the $x$ and $y$ spatial directions, respectively, a Poisson problem is solved by four matrix-vector multiplications and one solution of a diagonal linear system. Two of the matrices involved in the matrixvector multiplications have a leading dimension $N_{x}$, that of the other two is $N_{y}$, and $\left(N_{x}+N_{y}\right)$ algebraic equations must be solved.

The advantage of the matrix-diagonalization technique compared with the direct algorithm, in which the leading dimension of the matrix is $N_{x} \times N_{y}$, is decisive for the performance of grid independence studies at different parameter values. Typically upwards of 100 collocation points have been used to resolve each spatial direction. Results for the basic flow in the Reynolds number range $R e \in\left[10^{2}, 10^{4}\right]$ have been obtained in the square lid-driven cavity and their comparison against benchmark calculations is entirely acceptable, with our results for the location of the vortex cores agreeing better with the Richardson-extrapolated data of Schreiber \& Keller (1983) than those of Ghia et al. (1982). Subsequently, the depth of the cavity was kept equal to unity and steady laminar basic flows were obtained at aspect ratios $A=0.5,1,2,3$ and 4 in the neighbourhood of the tips of the neutral loops pertaining to each aspect ratio value.

\subsection{The ' $2 D C-L D C$ ' flow}

Finally, a fourth class of flows is identified by taking $\phi \in(0, \pi / 2)$ and considering solutions to the equations of motion in line with the assumption $\partial \overline{\boldsymbol{q}} / \partial z \equiv 0$, which will be discussed in the next section. In this manner a configuration combining the two-dimensional Couette (2DC) and the lid-driven cavity (LDC) basic flow is obtained. The basic (steady-state) flows $\overline{\boldsymbol{q}}=(\bar{u}, \bar{v}, \bar{w}, \bar{p})^{\mathrm{T}}$ possess a velocity vector with three non-zero velocity components; these can be obtained by solving (2.7)-(2.8) in addition to

$$
\frac{\partial \bar{w}}{\partial t}+\left\{\frac{\partial \psi}{\partial y} \frac{\partial \bar{w}}{\partial x}-\frac{\partial \psi}{\partial x} \frac{\partial \bar{w}}{\partial y}\right\}-\frac{1}{R e} \nabla_{2 d}^{2} \bar{w}=0,
$$

subject to the boundary conditions

$$
\begin{aligned}
\bar{u}(x, y=1) & =\cos \phi, \\
\bar{u}(x, y=0) & =\bar{u}(x=0, y)=\bar{u}(x=1, y)=0, \\
\bar{v}(x, y=0) & =\bar{v}(x, y=1)=\bar{v}(x=0, y)=\bar{v}(x=1, y)=0, \\
\bar{w}(x, y=1) & =\sin \phi, \\
\bar{w}(x, y=0) & =\bar{w}(x=0, y)=\bar{w}(x=1, y)=0 .
\end{aligned}
$$

Solutions to the system (2.7)-(2.9) are obtained numerically, using the spectral algorithm of the previous subsection. It is noted that at steady state solutions to (2.9) may be obtained in a decoupled manner from those of (2.7)-(2.8), such that the latter equations are first solved subject to (2.10)-(2.12); subsequently, (2.9) is solved subject to $(2.13)-(2.14)$. The limiting case $\phi=\pi / 2$ corresponds to the basic flow $\overline{\boldsymbol{q}}=(0,0, \bar{w}, \bar{p})^{\mathrm{T}}$ with $\bar{w}$ described by $(2.5)$, while that obtained at $\phi=0$ is the LDC basic flow $\overline{\boldsymbol{q}}=(\bar{u}, \bar{v}, 0, \bar{p})^{\mathrm{T}}$ discussed in the previous section. The gradual departure of the basic flow from the symmetric state described by (2.5) at the $\phi=\pi / 2$ limit may be seen in the dependence of $\bar{w}(x, y)$ on $\phi$ at $R e=100$ in figure 2 . 

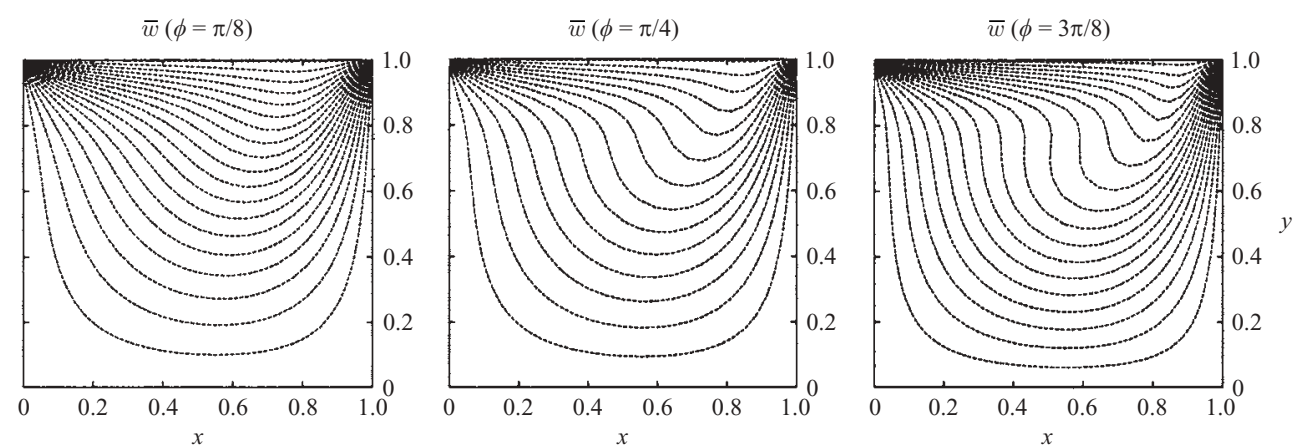

FIGURE 2. Dependence of $\bar{w}(x, y)$ on $\phi$ at $R e=100$ visualized in terms of 20 isolines drawn between the respective minimum and maximum values at $\phi=\pi / 8, \pi / 4$ and $3 \pi / 8$.

\section{Global linear stability analysis}

\subsection{Theory}

The present non-parallel linear stability analysis is concerned with flows which are homogeneous in only one of the three spatial directions. Conceptually, the class of problem which may be addressed in this framework is considerably broader than that to which classical linear theory applies (Tollmien 1929; Drazin \& Reid 1981; Schmid $\&$ Henningson 2001). Within the framework of the present analysis a solution of the equations of motion $Q$ is decomposed into the form

$$
\boldsymbol{Q}(x, y, z, t)=\overline{\boldsymbol{q}}(x, y)+\varepsilon \tilde{\boldsymbol{q}}(x, y, z, t)
$$

with $\overline{\boldsymbol{q}}=(\bar{u}, \bar{v}, \bar{w}, \bar{p})^{\mathrm{T}}$ indicating a steady two-dimensional basic flow, $(\bar{u}, \bar{v}, \bar{w})^{\mathrm{T}}$ and $\bar{p}$ denoting basic flow pressure. Superimposed upon $\overline{\boldsymbol{q}}$ are unsteady perturbations $\tilde{\boldsymbol{q}}$ with amplitude $\varepsilon \ll 1$. The decomposition (3.1) is substituted into the governing equations. At $O(1)$ the two-dimensional basic-flow terms satisfy the equations of motion and are consequently omitted from the resulting system, while quadratic terms in $\varepsilon$ are neglected. Clearly, if a numerically obtained basic flow does not satisfy exactly the equations of motion at $O(1)$, in terms of either poor spatial resolution or inadequate convergence in time, the associated eigenvalue problem results will be unreliable. Care must therefore be taken regarding the quality of numerically obtained basic flows; although modern tools for the numerical solution of the two-dimensional equations of motion have matured sufficiently for this problem to be considered closed (Karniadakis \& Sherwin 1999; Deville, Fischer \& Mund 2002), additional caution is warranted when solving for the basic flow of a global instability analysis, compared with the situation in classic linear theory.

At $O(\varepsilon)$ all terms are independent of the spatial coordinate $z$ and time $t$ and so an eigenmode Ansatz may be introduced, of the form

$$
\tilde{\boldsymbol{q}}(x, y, z, t)=\hat{\boldsymbol{q}}(x, y) \exp (\mathrm{i} \Theta)+\text { c.c. }
$$

where $\Theta=\beta z-\omega t$. Complex conjugation is introduced in (3.2), since all three of $\hat{\boldsymbol{q}}, \beta$ and $\omega$ can in general be complex, while $\tilde{\boldsymbol{q}}$ is real. In the temporal framework, as considered here, $\beta$ is taken to be a real and prescribed wavenumber parameter and $\omega$ is the corresponding complex eigenvalue (to be determined). In the most general case of a basic flow with three non-zero velocity components the following system of equations for the determination of the eigenvalue $\omega$ and the amplitude functions 
$\hat{\boldsymbol{q}}=(\hat{u}, \hat{v}, \hat{w}, \hat{p})^{T}$ results:

$$
\begin{aligned}
{\left[\mathscr{L}-\left(\mathscr{D}_{x} \bar{u}\right)\right] \hat{u}-\left(\mathscr{D}_{y} \bar{u}\right) \hat{v}-\mathscr{D}_{x} \hat{p} } & =-\mathrm{i} \omega \hat{u}, \\
-\left(\mathscr{D}_{x} \bar{v}\right) \hat{u}+\left[\mathscr{L}-\left(\mathscr{D}_{y} \bar{v}\right)\right] \hat{v}-\mathscr{D}_{y} \hat{p} & =-\mathrm{i} \omega \hat{v}, \\
-\left(\mathscr{D}_{x} \bar{w}\right) \hat{u}-\left(\mathscr{D}_{y} \bar{w}\right) \hat{v}+\mathscr{L} \hat{w}-\mathrm{i} \beta \hat{p} & =-\mathrm{i} \omega \hat{w}, \\
\mathscr{D}_{x} \hat{u}+\mathscr{D}_{y} \hat{v}+\mathrm{i} \beta \hat{w} & =0 .
\end{aligned}
$$

Here $\mathscr{L} \equiv(1 / R e)\left(\mathscr{D}_{x}^{2}+\mathscr{D}_{y}^{2}-\beta^{2}\right)-\bar{u} \mathscr{D}_{x}-\bar{v} \mathscr{D}_{y}-\mathrm{i} \beta \bar{w}, \mathscr{D}_{x}=\partial / \partial x, \mathscr{D}_{y}=\partial / \partial y$ and $R e$ is a suitably defined Reynolds number.

The governing equations for all four problems considered herein may be derived from (3.3)-(3.6). In both the pressure-gradient-driven rectangular duct flow and the wall-bounded Couette flow the only basic flow velocity component is $\bar{w}$, directed along the wavenumber direction. In this case (3.4)-(3.6) simplify to

$$
\begin{aligned}
\mathscr{L}_{1} \hat{u}-\mathscr{D}_{x} \hat{p} & =-\mathrm{i} \omega \hat{u}, \\
\mathscr{L}_{1} \hat{v}-\mathscr{D}_{y} \hat{p} & =-\mathrm{i} \omega \hat{v}, \\
-\left(\mathscr{D}_{x} \bar{w}\right) \hat{u}-\left(\mathscr{D}_{y} \bar{w}\right) \hat{v}+\mathscr{L}_{1} \hat{w}-\mathrm{i} \beta \hat{p} & =-\mathrm{i} \omega \hat{w}, \\
\mathscr{D}_{x} \hat{u}+\mathscr{D}_{y} \hat{v}+\mathrm{i} \beta \hat{w} & =0 .
\end{aligned}
$$

Here $\mathscr{L}_{1} \equiv(1 / R e)\left(\mathscr{D}_{x}^{2}+\mathscr{D}_{y}^{2}-\beta^{2}\right)-\mathrm{i} \beta \bar{w}$. This formal simplification does not translate into a reduction of the computing effort required for the solution of the partial derivative eigenvalue problem since (3.7)-(3.10) is also a complex system of the same size as the original problem (3.4)-(3.6). One algorithmic simplification that is possible in the case of a single basic-flow velocity component $\bar{w}$ along the wavenumber direction has been followed by Tatsumi \& Yoshimura (1990) who wrote (3.7)-(3.10) as a system of two partial differential equations, namely the generalized Orr-Sommerfeld and Squire system. The advantage of this approach is that the available computing resources can be devoted to the resolution of two, as opposed to four, coupled equations, thus achieving substantially higher resolution per eigenfunction than that possible when using (3.7)-(3.10). The disadvantage is that derivatives of fourth order in both of the two spatial directions and mixed derivatives of fourth order appear, which need a higher number of collocation points to be described adequately, compared with the second-order derivatives appearing in (3.7)-(3.10). Because of the latter reason we solved the eigenvalue problem in its form of the system (3.7)-(3.10). An additional difference between the approach of Tatsumi \& Yoshimura (1990) and that followed herein is that the former investigators took advantage of the symmetries existing in the basic flow to consider different classes of permissible solutions separately, which permits the solution of the instability problem on a quarter or half of the domain. Although this offers decisive efficiency advantages compared with the approach followed herein, these simplifications have not been invoked here in order to retain the ability to address the possibility of modes for which no such symmetries exist (indeed there are of course no such symmetries in the case of lid-driven cavity flows). In this manner we recover the symmetries of the eigenvalue problem (when present) as a result of our calculations, which offers additional validation of the algorithm.

However, one very useful simplification is possible without loss of generality in the computationally very demanding case of LDC flow. In this case the basic flow vector is $(\bar{u}, \bar{v}, 0, \bar{p})^{\mathrm{T}}$, and so no preferential direction exists in the spanwise direction $z$ such that the eigenvalue spectrum must be composed of either stationary disturbances or pairs of disturbances travelling in opposite $z$-directions. This physical fact may be exploited by writing (3.3)-(3.6) as a real coefficient eigenvalue problem, by simply 
redefining

$$
\beta \leftarrow \mathrm{i} \beta, \omega \leftarrow \mathrm{i} \omega
$$

which has the significant algorithmic implication of halving the storage requirements compared with those of the original problem; we emphasize here that this is not valid for the other three classes of basic flows. The real eigenvalue problem that was solved for the LDC flows was

$$
\begin{aligned}
{\left[\mathscr{L}_{2}-\left(\mathscr{D}_{x} \bar{u}\right)\right] \hat{u}-\left(\mathscr{D}_{y} \bar{u}\right) \hat{v}-\mathscr{D}_{x} \hat{p} } & =-\omega \hat{u}, \\
-\left(\mathscr{D}_{x} \bar{v}\right) \hat{u}+\left[\mathscr{L}_{2}-\left(\mathscr{D}_{y} \bar{v}\right)\right] \hat{v}-\mathscr{D}_{y} \hat{p} & =-\omega \hat{v}, \\
\mathscr{L}_{2} \hat{w}+\beta \hat{p} & =-\omega \hat{w}, \\
\mathscr{D}_{x} \hat{u}+\mathscr{D}_{y} \hat{v}-\beta \hat{w} & =0,
\end{aligned}
$$

where $\mathscr{L}_{2} \equiv(1 / \operatorname{Re})\left(\mathscr{D}_{x}^{2}+\mathscr{D}_{y}^{2}-\beta^{2}\right)-\bar{u} \mathscr{D}_{x}-\bar{v} \mathscr{D}_{y}$.

For all four closed-system problems considered, the boundary conditions $\hat{u}=\hat{v}=\hat{w}=0$ are imposed on the perturbation velocity components at the walls. Boundary conditions for the disturbance pressure do not exist physically; instead on the boundaries we collocate the compatibility conditions

$$
\begin{aligned}
& \frac{\partial \hat{p}}{\partial x}=\frac{1}{R e} \nabla_{2 d}^{2} \hat{u}-\bar{u} \frac{\partial \hat{u}}{\partial x}-\bar{v} \frac{\partial \hat{u}}{\partial y}, \\
& \frac{\partial \hat{p}}{\partial y}=\frac{1}{R e} \nabla_{2 d}^{2} \hat{v}-\bar{u} \frac{\partial \hat{v}}{\partial x}-\bar{v} \frac{\partial \hat{v}}{\partial y},
\end{aligned}
$$

derived from the Navier-Stokes equations at the boundary of the rectangular domains.

\subsection{Algorithmic considerations}

The characteristics of our algorithm are highlighted by contrasting them with the highorder numerical approaches in the literature, namely those of Tatsumi \& Yoshimura (1990) and Ding \& Kawahara (1998b). Compared with the spectral algorithm of Tatsumi \& Yoshimura (1990), the first difference is that we use a primitive variable formulation in order to facilitate the study of non-zero basic flow velocity components in more than one spatial direction; in this way a wider class of problems may be studied, including that in the lid-driven cavity. As has been mentioned a further advantage of the primitive variable formulation is that only first derivatives of the basic flow and up to second derivatives of the eigenfunctions appear; low-order derivatives are less sensitive to numerical discretization errors at modest resolution. The second difference (for the reasons mentioned above) is that we use the full domain on which general boundary conditions may be specified. Third, unlike the traditionally used Chebyshev or Legendre polynomials we use an algorithm based on arbitrary Jacobi collocation which permits spectrally accurate resolution of instability modes with significant gradients away from the wall (also termed 'centre-modes' in the context of channel flow instability). The ability to place grid points in an optimal manner offers a significant advantage over Legendre (or Chebyshev) collocation in resolving any such modes that might occur, at reasonable computing cost. The power of spectral methods to deliver results of high accuracy compared with finitedifference discretizations at a fixed maximally affordable resolution, is fully utilized in this type of problem. We also note that our code is shielded from the details of the spectral basis functions and it is straightforward to interchange the latter at will for diagnostic purposes before an optimum choice is made for production runs. Fourth, high resolution demands are placed on the stability calculations in order to resolve the need for large numbers of grid points in high Reynolds number 
cases and also in cases of large aspect ratio. For these problems the QZ algorithm employed by Tatsumi \& Yoshimura (1990) is inappropriate since the memory and runtime requirements scale with the square and the cube of the number of discretization nodes, respectively. The inverse Rayleigh iteration (Wilkinson 1965) is also not practical since, like the QZ algorithm, it requires storage of auxiliary matrices. Instead, we use the Arnoldi algorithm (Saad 1980) which delivers a number of the eigenvalues in the neighbourhood of a specified estimate (usually in the vicinity of the unstable/least-stable eigenvalue) at a small fraction of the cost pertinent to an equivalent QZ or inverse Rayleigh procedure. In a first step either (3.7)-(3.10) or (3.12)-(3.15) are written in the symbolic form

$$
\boldsymbol{A}=\omega \boldsymbol{B},
$$

with the boundary conditions incorporated. The Arnoldi algorithm may be seen as a conceptual link between the approaches of inverse Rayleigh iteration, which delivers the most unstable eigenvalue, and the QZ algorithm which recovers the full eigenvalue spectrum. By contrast, the Arnoldi algorithm delivers not only the physically interesting most-unstable/least-stable eigenmode but also a number of other eigenvalues in the neighbourhood of the specified target eigenvalue. The number of eigenvalues delivered is determined by the freely chosen subspace dimension, the limit of which is the leading dimension of matrices $\boldsymbol{A}$ and $\boldsymbol{B}$. A so-called shift-andinvert strategy is also implemented in our eigenvalue problem solver, according to which instead of (3.18) one solves

$$
\hat{\boldsymbol{A}} X=\mu X, \quad \hat{\boldsymbol{A}}=(\boldsymbol{A}-\sigma \boldsymbol{B})^{-1} \boldsymbol{B}, \quad \mu=\frac{1}{\omega-\sigma} .
$$

Use of this technique, combined with the special structure of $\boldsymbol{B}$ in (3.18) results in the need to store just one matrix, a modified version of the LU-decomposition of $\boldsymbol{A}$. For matrices of size of several gigabytes, the ability to store one (in the Arnoldi algorithm) instead of four (in the QZ or the inverse Rayleigh iteration algorithms) gives the former algorithm a decisive efficiency advantage compared with the latter classic algorithms. In this respect we note that the Arnoldi algorithm introduces an additional parameter to be specified, namely the estimated eigenvalue $\sigma$ in the neighbourhood of which the spectrum of the original problem is to be resolved. Further parametric studies must therefore be performed in order for the results obtained to be independent of this shift parameter. We note also that, while it is difficult to compare aspects of accuracy and efficiency between ours and the algorithm of Ding \& Kawahara (1998b) since these authors use a finite-element methodology as opposed to the spectral collocation used herein, both approaches rely on Krylov subspace iteration methods. One recent successful application of the algorithms employed herein for the solution of the most-general form of the global eigenvalue problem (3.3)-(3.6) has been presented by Theofilis et al. (2003).

\section{The stability analyses}

\subsection{The rectangular duct}

In this section we revisit the rectangular duct flow whose global linear instability was originally studied by Tatsumi \& Yoshimura (1990) and present some algorithmic aspects. Results were obtained using resolutions up to $\left(N_{x}, N_{y}\right)=(72,40)$ and a constant Krylov subspace dimension of 400. The coupled resolution of two spatial directions results in a matrix of leading dimension up to $1.1 \times 10^{4}$. Convergence studies 


$\begin{array}{rrrrr}A & R e & \beta & \omega_{\mathrm{r}} \\ 3.5 & 36600 & 0.71 & 0.12353 \\ 4.0 & 18400 & 0.80 & 0.16187 \\ 5.0 & 10400 & 0.91 & 0.21167 \\ 6.0 & 8200 & 0.94 & 0.22925 \\ 8.0 & 6800 & 0.98 & 0.24963 \\ 25.0 & 5772 & 1.02 & 0.26960 \\ \text { TABLE 2. Critical frequencies } \omega_{\mathrm{r}} \text { of mode I (Tatsumi \& Yoshimura 1990) as a function of the } \\ \text { duct aspect ratio } A .\end{array}$

were performed using resolutions up to the maximum of the available memory on the hardware utilized, 6 Gbytes (the array $\hat{\boldsymbol{A}}$ in (3.19) is kept in core memory). The fundamental symmetries expected in the eigenfunctions were recovered, and good agreement was observed with the results of Tatsumi \& Yoshimura (1990).

A naturally arising question of interest is whether use of this more general algorithm permits the appearance of modes other than those reported by Tatsumi \& Yoshimura (1990) to appear in the global eigenspectrum. This question has been pursued by a systematic examination of the instability of the flow using $R e \in[1000,50000]$ and $\beta=0(0.25) 6$. In these ranges we were unable to find any unstable disturbance other than those reported by Tatsumi \& Yoshimura (1990). For the sake of completing the information presented in the latter work, we have followed mode I and monitored its critical conditions as a function of the duct aspect ratio. The critical frequency results obtained are presented in table 2; the corresponding growth rates are less than $10^{-5}$. The conclusion of this part of our work is that despite the use of a more general algorithm for the solution of the partial-derivative eigenvalue problem and extensive numerical experimentation the results presented by Tatsumi \& Yoshimura (1990) have been found to be the only linear instabilities in the rectangular duct flow. A corollary of this is that the rectangular duct is more stable than its PPF idealized counterpart and the question of subcritical instability in the duct remains open.

\subsubsection{The effect of lateral walls on the spectrum of plane Poiseuille flow}

In the limit $A \rightarrow \infty$ the basic flow (2.3) merges into the PPF parabolic profile and one interesting question is the relationship between the eigenvalue spectra of pressuregradient-driven rectangular duct flow and PPF. This question was not addressed by Tatsumi \& Yoshimura (1990) who instead recovered the Orr-Sommerfeld and Squire spectra by simply neglecting the flow derivatives in the lateral spatial direction. Here we attempt to relate the two spectra in a more formal, continuous manner by permitting the duct aspect ratio to attain large finite values while resolving both inhomogeneous spatial directions.

In order to address this question we reverted to the QZ algorithm for the calculation of the entire flow eigenspectrum. Since storage of four matrices is required the attainable resolution is decreased considerably in comparison with the results presented in the previous section. Available hardware has limited resolution to $N_{x} \times N_{y}=2^{11}$ Legendre collocation points in all subsequent runs, calling for 3.6 Gbytes core memory and $O\left(10^{4}\right)$ CPU seconds runtime at 2.5 Gflops for the recovery of the entire eigenspectrum at a single $(R e, \beta)$ pair. We have lowered the Reynolds number values to $R e=\left[10^{2}, 10^{3}\right]$, kept a constant $\beta=1$ and examined flows in ducts having $A \in[1,100]$. All recovered eigenvalues at this Reynolds number value 


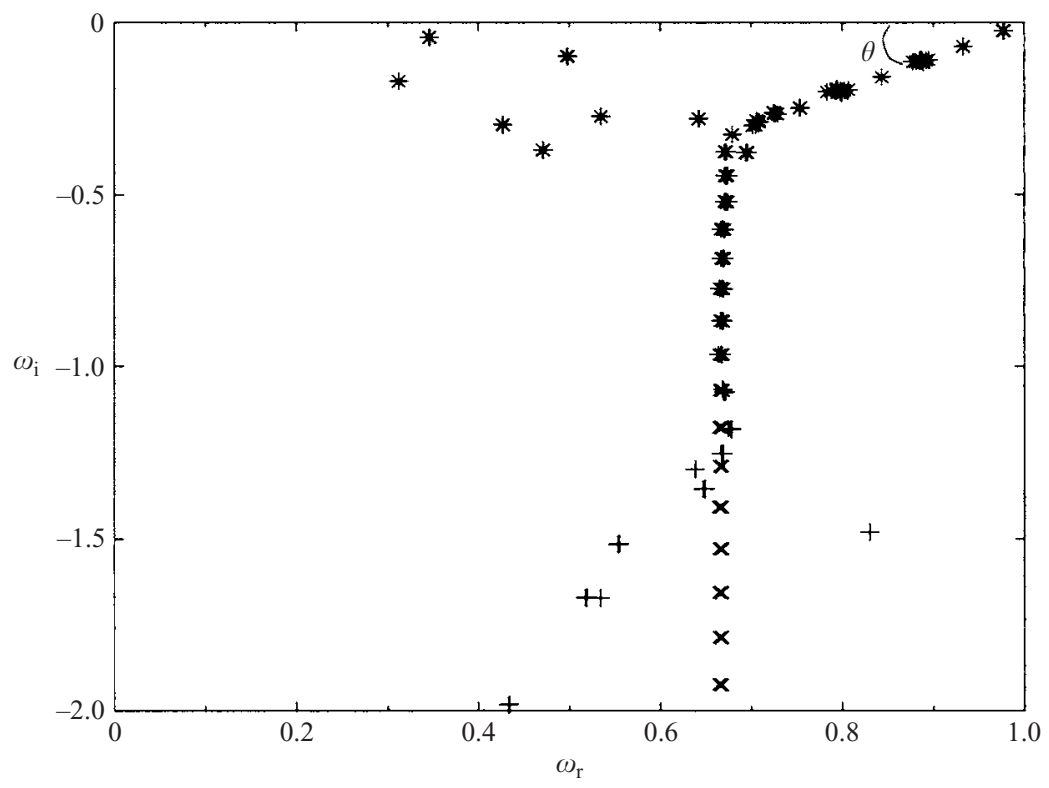

FIGURE 3. The least-stable part of the PPF eigenspectrum recovered using $N_{y}=40(+)$ and $64(\times)$ collocation points to resolve the $y$-direction.

correspond to stable disturbances; without entering into a detailed discussion of either issue here, we justify interest in stable eigenvalues from a physical point of view on account of the subcritical instability of the rectangular duct flow, as well as the potential role of the eigenspectrum in the framework of transient growth studies (e.g. Reddy et al. 1998) which are yet to be performed in these rectangular ducts.

The results to be presented were confirmed by higher resolution runs using the Arnoldi algorithm and $N_{x} \times N_{y}=2^{12}$ points, which necessitates 5.6 Gbytes core memory and $5 \times 10^{3} \mathrm{CPU}$ seconds runtime at 3.6 Gflops. From a numerical point of view, the modest resolution used in the QZ runs permits recovery of only a small part of the least-stable eigenvalue spectrum using 64-bit arithmetic. This well-known result (Dongarra, Straughan \& Walker 1996) is highlighted in the present partialderivative eigenvalue problem context in figure 3 . Here the PPF linear eigenspectrum is recovered at $R e=10^{3}, \beta=1$ by permitting $A \rightarrow \infty$, in practice taking $A=100$. Since we solve the linearized Navier-Stokes and continuity equations, rather than the single Orr-Sommerfeld equation, both the Orr-Sommerfeld and the Squire spectra are to be found in this result. The key point here is that the least-stable part of the PPF eigenspectrum can be recovered accurately from (3.6)-(3.5) in the limit $|\partial \overline{\boldsymbol{q}} / \partial x| \ll|\partial \overline{\boldsymbol{q}} / \partial y|$, including the prediction of the angle $\theta=\pi / 4$ between the axis $\omega_{\mathrm{i}}=0$ and the P-family of eigenmodes (Mack 1976); in other words the global eigenvalue spectrum is found to merge into that delivered by the classic linear theory at the appropriate limit. The numerical artifact of splitting of the $\left(\omega_{\mathrm{r}}=2 / 3, \omega_{\mathrm{i}} \rightarrow-\infty\right)$ part of the eigenspectrum in the low-resolution result is also shown in figure 3 . An increase of $N_{x}$ does not alter this result; rather $N_{x}$ copies of the respective PPF eigenvalues are recovered. It is an increase in $N_{y}$ which delivers additional $\omega_{\mathrm{i}} \rightarrow-\infty$ eigenvalues on the $\omega_{\mathrm{r}}=2 / 3$ line.

Figure 4 shows the effect of a variable aspect ratio on the least-stable part of the eigenspectrum for $A=1,2,3,4,5,10$, using a resolution of $\left(N_{x}, N_{y}\right)=(32,64)$ 
(a)
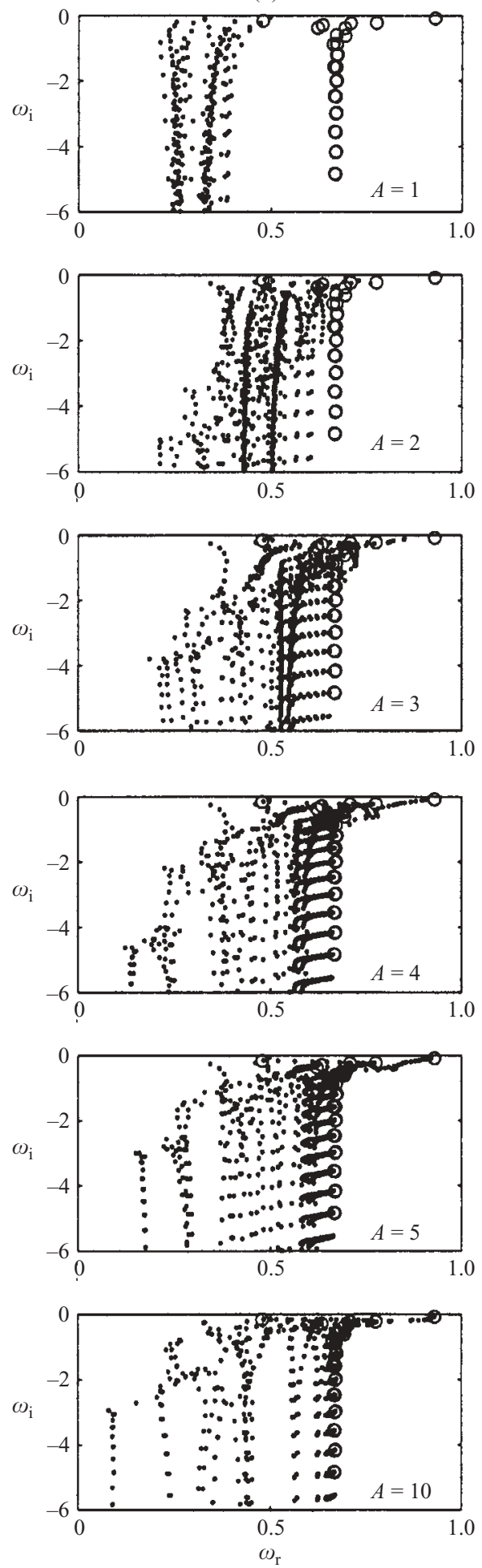

(b)
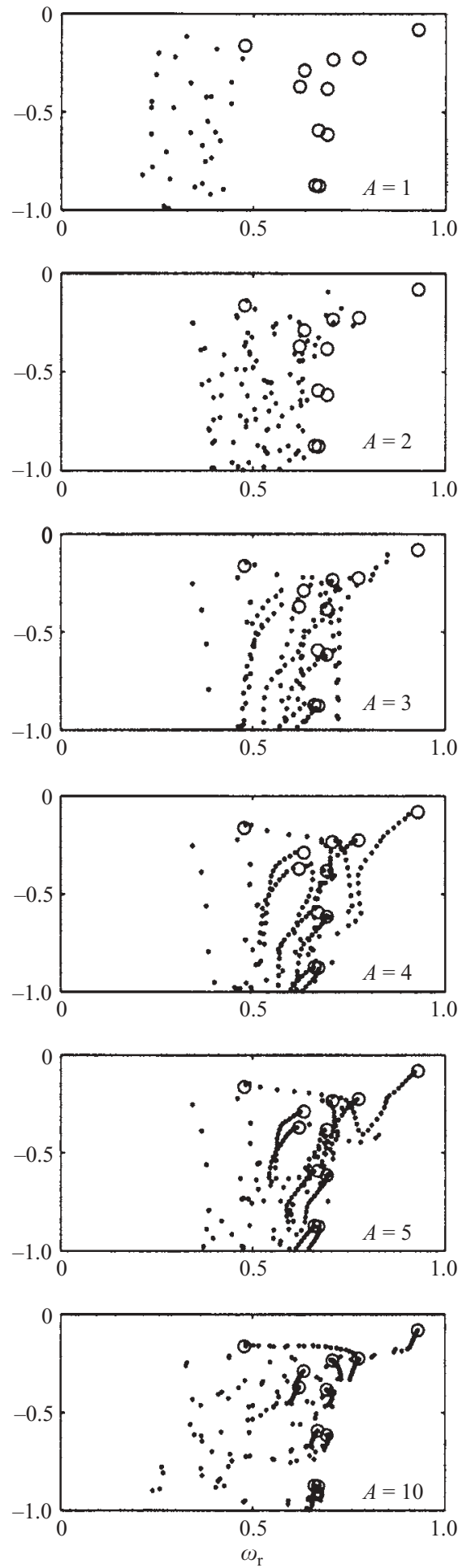

FIGURE 4. (a) The shifting of the least-stable part of the rectangular duct eigenspectrum with decreasing aspect ratio $A$, obtained using a resolution $(N x, N y)=(32,64)$ Legendre collocation points at $R e=100$. (a) Detail of the same result near the junction of the eigenspectrum branches. The open symbols show the corresponding PPF eigenspectrum. 


\begin{tabular}{ccccccc} 
& & & \multicolumn{4}{c}{$\sigma=1.0$} \\
\cline { 3 - 7 } Resolution & DOF $\times 10^{3}$ & Size $(\mathrm{Mb})$ & $\omega_{\mathrm{r}}$ & $\omega_{\mathrm{i}}$ & $\omega_{\mathrm{r}}$ & $\omega_{\mathrm{i}}$ \\
$20 \times 20$ & 1.6 & 48 & 0.9021 & -0.0624 & 0.9001 & -0.0716 \\
$29 \times 29$ & 3.3 & 202 & 0.9031 & -0.0622 & 0.8990 & -0.0696 \\
$40 \times 40$ & 6.4 & 630 & 0.9033 & -0.0622 & 0.8991 & -0.0703 \\
$57 \times 57$ & 13.0 & 2730 & 0.9033 & -0.0622 & 0.8991 & -0.0703
\end{tabular}

TABLE 3. Convergence studies in wall-bounded Couette flow at $A=2, R e=3800, \beta=1$. Shown are the two least-stable eigenvalues $\left(\omega_{\mathrm{r}}, \omega_{\mathrm{i}}\right)$ in the neighbourhood of $\sigma=1.0$ as functions of resolution of the two spatial directions and total number of degrees of freedom (DOF).

Legendre collocation points. Point symbols denote the eigenspectrum of the rectangular duct and open symbols have been used for the PPF eigenspectrum, which has been superimposed on the former for comparison. Resolution studies (not presented here) confirm the integrity of these results. Some observations worthy of mention are the following. In addition to the PPF branch $\left(\omega_{\mathrm{r}}=2 / 3, \omega_{\mathrm{i}} \rightarrow-\infty\right)$, other branches with $\left(\omega_{\mathrm{r}}=\right.$ const, $\left.\omega_{\mathrm{i}} \rightarrow-\infty\right)$ are introduced on account of the lateral walls of the duct. The available resolution does not permit a definitive statement regarding the values of the respective constant frequency values but what can be confidently stated is that all duct branches have

$$
\omega_{\mathrm{r}} \leqslant 2 / 3
$$

i.e. the frequency of the PPF S-family represents an upper bound for all rectangular duct modes which pertain to the newly found rectangular-duct S-families. At the low resolution of the $x$-direction the additional branches are already present while their number increases when resolution of this direction is increased. A hint regarding the origin of the additional branches is given by the large- $A$ results. It can be seen in figure 4 that several of the duct modes emanate from their PPF counterparts, with clusters of duct modes clearly being associated with a single PPF eigenvalue. No doubt on account of the marginal resolution, the picture becomes increasingly blurred as $A \rightarrow 1$; what does become clear in this limit is that several duct modes cluster into two additional branches of the P family. Further quantification of these qualitative observations is necessary but cannot be undertaken on present-generation hardware.

\subsection{Wall-bounded Couette flow}

In a manner analogous to that presented in the previous section, we investigate the limit of large aspect ratio in order to connect our results with eigenmodes of the one-dimensional flow. A large number of runs were performed in finite-aspect-ratio ducts; without prior guidance regarding regions of potential instability in this flow we have first established adequacy of resolution and associated subspace dimension at a given Reynolds number. In table 3 we present convergence history results at $A=2, R e=3800, \beta=1$ using a subspace dimension $m=200$. We have sampled the domain $\sigma \in[0, A]$ and present the two least-stable eigenvalues in the neighbourhood of two different locations in parameter space, $\sigma=0.5$ and 1 ; results identical to those at $\sigma=1$ were obtained when using a shift parameter $\sigma=2$, suggesting that the eigenvalue spectrum of the wall-bounded Couette flow is also confined in $\omega_{\mathrm{r}} \in[-1,1]$. Further observations made are the following. As $\omega_{\mathrm{r}} \rightarrow 1$ we find that $\omega_{\mathrm{i}} \uparrow 0$ which results in easier resolution of the spectrum in the neighbourhood of $\omega_{\mathrm{r}}=1$ at a given 
discretization compared with that near $\left|\omega_{\mathrm{r}}\right| \ll 1$. One obvious reason for this is that the eigenspectrum appears to be composed of increasingly stable modes as $\omega_{\mathrm{r}} \rightarrow 0$ in a manner analogous to the Y-shaped PCF eigenspectrum. A more subtle reason related to the difficulty of resolving the neighbourhood of $\omega_{\mathrm{r}}=0$ in the spectrum has been extensively discussed in the context of the stability of the linear profile by Dongarra et al. (1996) and is related to the finite precision at which computations are performed. However, the size of the matrices of the two-dimensional eigenvalue problem prohibit use of 128-bit arithmetic in the context of the present calculations.

From a physical point of view the results of table 3, which are typical of results obtained at different aspect ratios, suggest that the least-stable part of the eigenspectrum is resolved well in the present calculations up to the highest Reynolds numbers examined, $R e \approx 5000$. Indeed, while resolutions in excess of $58^{2}$ collocation points are still inadequate to recover a converged eigenvalue in the neighbourhood of $\sigma=0.5$ or lower, $30^{2}$ points suffice to yield two converged decimal places at $\sigma=1$. Calculations performed using high Krylov subspace dimension have demonstrated that the analogue of the well-known Y-shaped eigenvalue spectrum of PCF also exists in the presence of lateral walls.

Compared with the infinite-aspect-ratio counterpart of this flow at $R e=3800, \beta=1$ (Dongarra et al. 1996) it can be seen that the resolved parts of the spectrum of the $A=2$ flow correspond to more strongly damped eigenmodes. This is an observation which has been repeated at all aspect ratios examined. In a large but finite number of calculations performed up to the highest Reynolds numbers that could be addressed numerically we have have consistently made two observations: first, no unstable modes have been found to exist in wall-bounded Couette flow and secondly a reduction of the aspect ratio from infinity to a finite value larger than unity has a stabilizing effect on the eigenspectrum of the PCF.

\subsection{The lid-driven cavity flow}

We obtained basic flow solutions on rectangular spectral collocation grids using high resolution inaccessible to the instability analysis and used a cubic spline interpolation scheme to transpose the basic flow solution on to the stability analysis grid. Care has been taken that the basic flow solutions be converged in time to within a tolerance $t o l \equiv\left|\left(f_{t_{0}+\Delta t}-f_{t_{0}}\right) / f_{t_{0}}\right|<10^{-12}$, where $f$ is an integral measure of the flow or the value of a local flow quantity, resulting at moderate Reynolds numbers $R e \sim O\left(10^{3}\right)$ in integration times $t_{0}>2000$ when initializing the basic flow solution from zero and $t_{0}>1000$ when using the converged solution at a neighbouring Reynolds number value to obtain the steady state at a new Reynolds number value.

\subsubsection{The square cavity}

We have first investigated a low Reynolds number value, $R e=200$, where the previously published sets of results are consistent. Only Ramanan \& Homsy (1994) have presented tabulated values of their results, while Ding \& Kawahara $(1998 a, b)$ have restricted themselves to graphical comparisons with the results of Ramanan \& Homsy (1994). In table 4 we show a comparison of our results with the tabulated and graphically reproduced results of the previous investigations; we see that the discrepancy between the previous and the present results is within that between the results of Ramanan \& Homsy (1994) and Ding \& Kawahara (1998b). We will return to the point of convergence of instability results shortly. In general, however, we notice a good qualitative and a reasonable quantitative agreement of the previous and the present instability analyses, which all confirm the experimentally established 


\begin{tabular}{|c|c|c|c|c|c|}
\hline \multirow[b]{2}{*}{$\beta$} & \multicolumn{2}{|c|}{ RH } & \multirow{2}{*}{$\begin{array}{c}\mathrm{DK} \\
\omega_{\mathrm{i}}\end{array}$} & \multicolumn{2}{|c|}{ Present results } \\
\hline & $\omega_{\mathrm{i}}$ & $\omega_{\mathrm{r}}$ & & $\omega_{\mathrm{i}}$ & $\omega_{\mathrm{r}}$ \\
\hline 1 & 0.34 & \pm 0.00 & 0.3183 & 0.3297 & \pm 0.0000 \\
\hline 2 & 0.23 & \pm 0.00 & 0.2248 & 0.2267 & \pm 0.0000 \\
\hline 3 & 0.29 & \pm 0.11 & 0.2924 & 0.2954 & \pm 0.1073 \\
\hline 4 & 0.30 & \pm 0.28 & 0.2969 & 0.2956 & \pm 0.2810 \\
\hline 5 & 0.34 & \pm 0.43 & 0.3431 & 0.3404 & \pm 0.4260 \\
\hline 6 & 0.39 & \pm 0.58 & 0.3893 & 0.3844 & \pm 0.5821 \\
\hline 7 & 0.41 & \pm 0.67 & 0.4073 & 0.4013 & \pm 0.6733 \\
\hline 8 & 0.45 & \pm 0.72 & 0.4637 & 0.4587 & \pm 0.7232 \\
\hline 9 & 0.54 & \pm 0.76 & 0.5504 & 0.5473 & \pm 0.7622 \\
\hline
\end{tabular}

TABLE 4. Comparison of the least-stable eigenmode at $R e=200$ to the results of Ramanan \& Homsy (1994) (RH) and the graphically (digitally) reproduced growth rate result of Ding \& Kawahara (1998b) (DK).
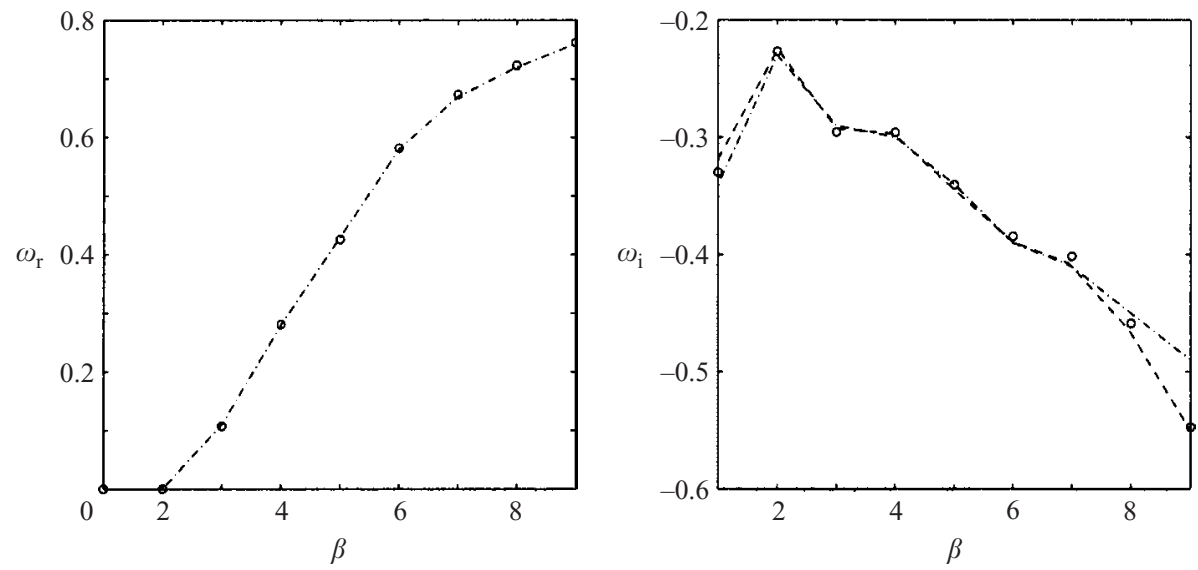

Figure 5. Comparison of the dependence of $\omega_{\mathrm{r}}$ and $\omega_{\mathrm{i}}$ on $\beta$ as obtained by Ramanan \& Homsy (1994) (dash-dotted line), Ding \& Kawahara (1998b) (dashed line) and the present results (symbol) at $R e=200$.

fact of stability of the two-dimensional basic flow at this Reynolds number. The same results are presented in graphical form in figure 5 for the dependence of the frequency $\omega_{\mathrm{r}}$ and the damping rates $\omega_{\mathrm{i}}$ (defined as having an opposite sign to that of the present work) on the spanwise wavenumber. While the frequency of this instability appears to be a smooth function of the wavenumber, the dependence of the damping rate on the wavenumber does not follow the familiar bell-shaped curve pattern; one possible explanation for this observation is that the results of table 4 and figure 5 do not correspond to a single eigenmode. We have verified this conjecture by reverting to the QZ algorithm while lowering the Reynolds number to $R e=100$ in order to ensure convergence. A very large number of computations were then performed to recover the full eigenvalue spectrum. Qualitatively the picture was found to be analogous to that described (in a different, but related context) by Stocker \& Duck (1995), in which modes continuously merge and split if one monitors the dependence of $\omega_{\mathrm{i}}$ on $\beta$, while that of $\omega_{\mathrm{r}}$ on $\beta$ exhibits a 'honeycomb' pattern. 


\begin{tabular}{|c|c|c|c|c|c|c|c|c|}
\hline \multirow[b]{2}{*}{ EVP Grid } & \multicolumn{2}{|c|}{$64^{2}$} & \multicolumn{4}{|c|}{ BF Grid } & \multicolumn{2}{|c|}{$160^{2}$} \\
\hline & $\omega_{\mathrm{i}}$ & $\omega_{\mathrm{r}}$ & $\omega_{\mathrm{i}}$ & $\omega_{\mathrm{r}}$ & $\omega_{\mathrm{i}}$ & $\omega_{\mathrm{r}}$ & $\omega_{\mathrm{i}}$ & $\omega_{\mathrm{r}}$ \\
\hline $32 \times 32$ & 0.0000 & \pm 0.4988 & 0.0006 & \pm 0.4974 & 0.0021 & \pm 0.4963 & 0.0037 & \pm 0.4961 \\
\hline $40 \times 40$ & 0.0041 & \pm 0.4985 & 0.0033 & \pm 0.4980 & 0.0034 & \pm 0.4986 & 0.0030 & \pm 0.4959 \\
\hline $48 \times 48$ & 0.0027 & \pm 0.4967 & 0.0017 & \pm 0.4992 & 0.0043 & \pm 0.4980 & 0.0046 & \pm 0.4976 \\
\hline $56 \times 56$ & 0.0039 & \pm 0.4951 & 0.0038 & \pm 0.4984 & 0.0043 & \pm 0.4981 & 0.0043 & \pm 0.4979 \\
\hline
\end{tabular}

TABLE 5. Convergence history of the least-stable eigenmode at $R e=900, \beta=7.35$, using a Krylov subspace dimension $m=400$ as function of the resolutions used for the basic flow (BF) and the eigenvalue problem (EVP).

\begin{tabular}{|c|c|c|c|c|c|c|c|c|}
\hline \multirow[b]{2}{*}{ EVP Grid } & \multicolumn{2}{|c|}{ S1 } & \multicolumn{2}{|c|}{$\mathrm{T} 1$} & \multicolumn{2}{|c|}{$\mathrm{T} 2$} & \multicolumn{2}{|c|}{$\mathrm{T} 3$} \\
\hline & $\omega_{\mathrm{i}}$ & $\omega_{\mathrm{r}}$ & $\omega_{\mathrm{i}}$ & $\omega_{\mathrm{r}}$ & $\omega_{\mathrm{i}}$ & $\omega_{\mathrm{r}}$ & $\omega_{\mathrm{i}}$ & $\omega_{\mathrm{r}}$ \\
\hline $16 \times 16$ & 0.3420 & \pm 0.0000 & 0.1280 & \pm 0.6651 & 0.0232 & \pm 0.4924 & 0.0958 & \pm 1.3140 \\
\hline $24 \times 24$ & 0.1426 & \pm 0.0000 & 0.1073 & \pm 0.6929 & 0.0064 & \pm 0.4976 & 0.1048 & \pm 1.3838 \\
\hline $32 \times 32$ & 0.1417 & \pm 0.0000 & 0.1084 & \pm 0.6936 & 0.0021 & \pm 0.4963 & 0.1071 & \pm 1.3832 \\
\hline $40 \times 40$ & 0.1420 & $\overline{ \pm} 0.0000$ & 0.1067 & $\overline{ \pm} 0.6924$ & 0.0034 & \pm 0.4986 & 0.1032 & \pm 1.3850 \\
\hline $48 \times 48$ & 0.1423 & \pm 0.0000 & 0.1071 & \pm 0.6929 & 0.0043 & \pm 0.4980 & 0.1045 & \pm 1.3848 \\
\hline $56 \times 56$ & 0.1425 & \pm 0.0000 & 0.1071 & \pm 0.6928 & 0.0043 & \pm 0.4981 & 0.1044 & $\begin{array}{l} \pm \\
\pm\end{array} .3846$ \\
\hline
\end{tabular}

TABLE 6. The four least-stable modes at $R e=900, \beta=7.35$.

We now turn our attention to a Reynolds number value $R e=900$, with a spanwise wavenumber $\beta=7.35$, which is supercritical according to Ramanan \& Homsy (1994), who predicted $R e_{\text {crit }} \approx 594$, subcritical to those reported by Ding \& Kawahara (1998a,b), $R e_{\text {crit }} \approx 1025$ and $R e_{\text {crit }} \approx 920$ respectively, and within the range of Reynolds numbers at which instability has observed experimentally by Aidun et al. (1991) and Benson \& Aidun (1992), $R e_{\text {crit }} \in$ [825,925]. At this Reynolds number we carefully monitored the convergence of the eigenvalue calculations with respect to both the basic flow and instability analysis grids. Table 5 indicates that for this particular test case, a basic flow grid of 128 points in both spatial dimensions is sufficient for up to four-decimal-place accuracy of the eigenvalue. A scan of the parameter range $R e \in[775,1100]$ and $\beta \in[0,30]$ was performed in increments of 25 in Reynolds number and 0.5 in wavenumber, at a total cost of $280 \mathrm{CPU}$ hours on a NEC SX-4 machine requiring up to 1.6 Gbytes of memory (excluding the cost of the calculation of the basic flows). In addition to the least-stable mode, a plethora of stable modes is recovered by the Arnoldi algorithm; a number of these are presented in table 6 . The mode found by Ding \& Kawahara (1998b) is denoted here by T2 and is indeed the least-damped eigenmode at these parameter values. The nomenclature and order of presentation of the additional modes will become apparent in what follows. At these conditions, however, the additional modes are more strongly damped than those discussed by Ding \& Kawahara (1998b) and consequently less interesting from the physical point of view. Note that a stability analysis grid composed of $48^{2}$ collocation points is seen to be adequate for the results to have converged to nearly four decimal places.

Further evidence of the integrity of our results is presented in figure 6 showing a comparison between the growth rates of mode T2, as determined by our calculation (indicated by a solid line), and the graphically reproduced results of Ding \& Kawahara 


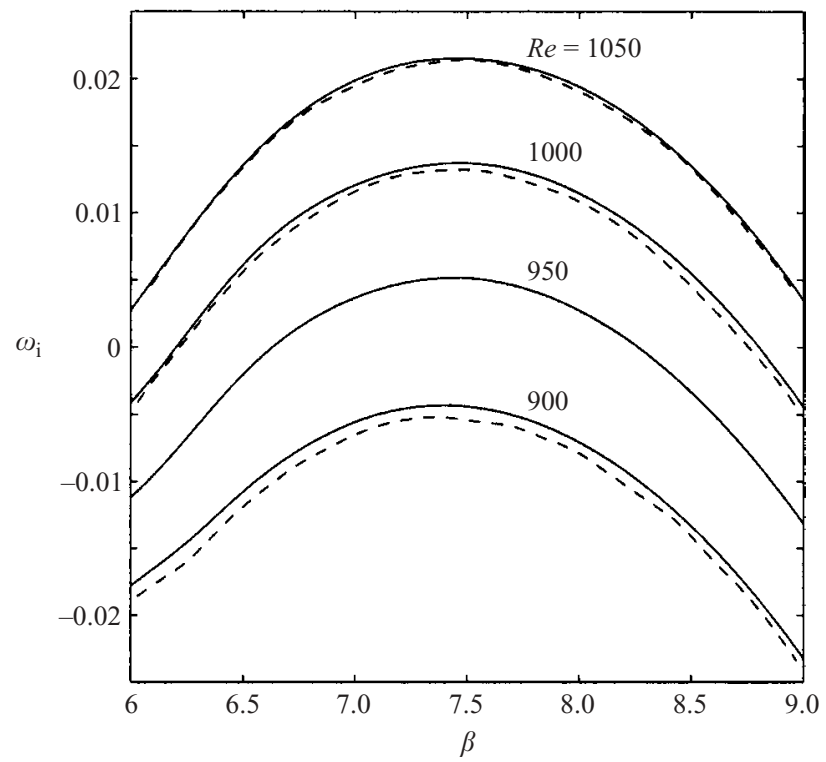

Figure 6. Dependence of the growth rate $\omega_{\mathrm{i}}$ on the wavenumber $\beta$ as obtained by Ding \& Kawahara (1998b) (dashed) and our calculations (solid).

(1998b), shown as dashed lines; the agreement is very good. In view of these results (and many other results computed but not reported here), further analyses of the square lid-driven cavity flow were generally obtained using a grid for the recovery of the basic flow comprising $128^{2}$ collocation points and a grid for the discretization of the global eigenvalue problem using $48^{2}$ collocation points per eigenfunction.

We now move on to consider neutral stability curves for the first four modes. These were obtained by first linearly interpolating between two data curves, namely that corresponding to the least-stable disturbances pertinent to each mode and that at the next higher Reynolds number calculated. Next, the resulting data were used to calculate the critical parameters, again by linear interpolation. The neutral stable curves are shown in figure 7(a). Here we have adopted the following notation: the stationary mode $\mathrm{S} 1$ is denoted by a circle, and the travelling modes T1, T2 and T3 by a square, diamond and triangle, respectively. The critical Reynolds numbers and wavenumbers $\beta$ for these first four modes are presented at aspect ratio $A=1$ in table 7 . The plot in figure $7(b)$ retains the notation introduced and shows a comparison of frequency $f \equiv \omega_{\mathrm{r}} / 2 \pi$ with experimental data over a range Reynolds numbers. Note that in computing these results we have varied $\beta$ in such a way as to predict the mostamplified/least-unstable modes. We see that our results for mode T1 compare very favourably with the travelling mode monitored in the experimental data of Benson \& Aidun (1992); at its largest, in the neighbourhood of $R e=1050, \beta=9$, the discrepancy between the experimental result and our prediction of the most unstable travelling mode is $O(0.7 \%)$. The present results on the square lid-driven cavity flow are in very good agreement with those presented by Theofilis (2000) and Albensoeder et al. $(2001 b)$, both of which have independently established the existence of three modes, one stationary (S1) and two travelling (T1 and T3), beyond that earlier reported by Ding \& Kawahara (1998b).

In figure 8 we present the spatial structure of the eigenfunctions of the four most unstable modes for the square lid-driven cavity at $R e=1000$. The respective 

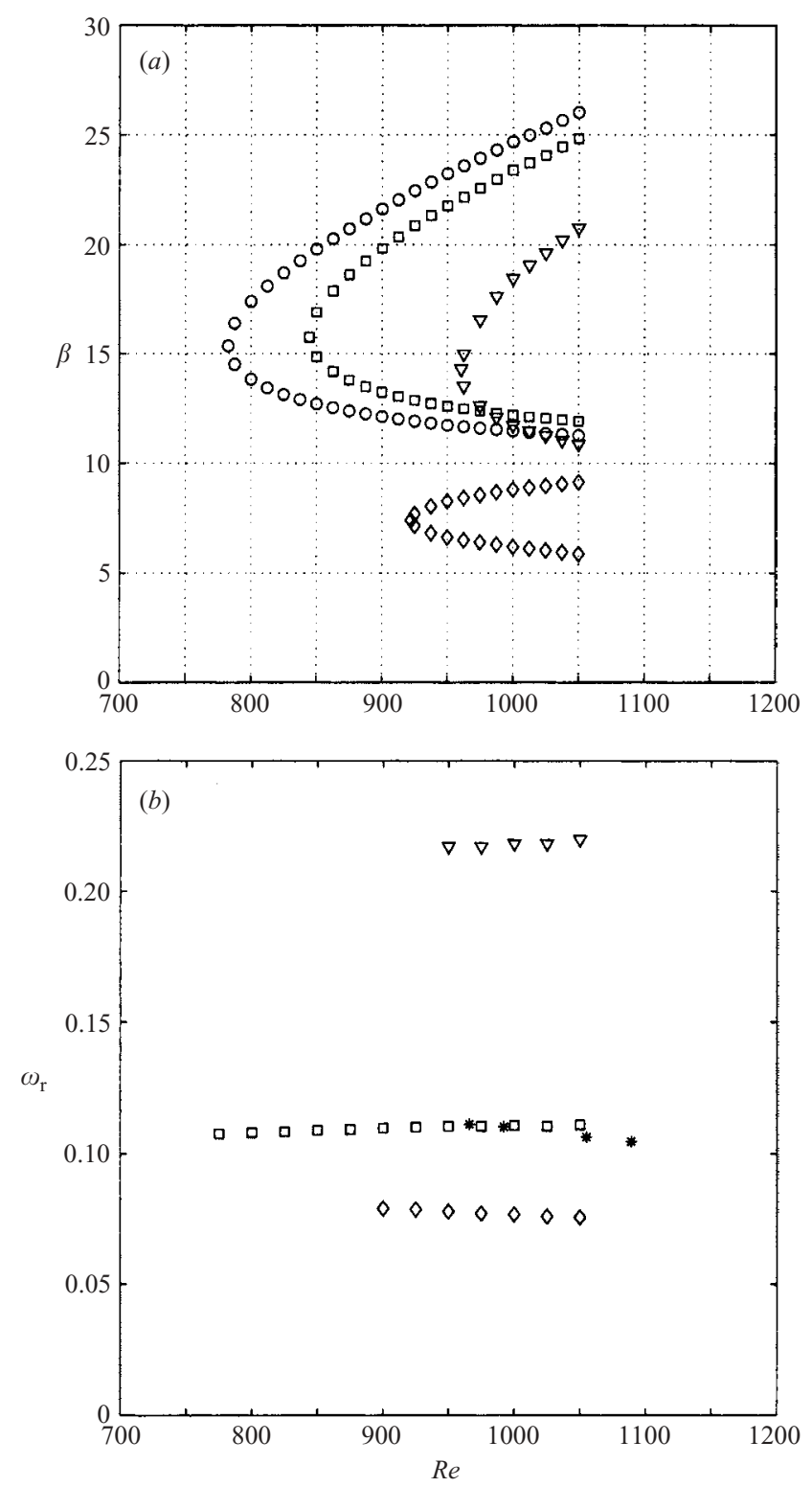

FIGURE 7. (a) Neutral curves of the first four eigenmodes in the square lid-driven cavity. Stationary mode S1 (circle); travelling modes T1 (square), T2 (diamond), T3 (triangle). (b) Frequencies of these eigenmodes compared with the experimental result of Benson \& Aidun (1992) (star symbol).

wavenumbers are $\beta=15$ for modes S1, T1 and T3 and $\beta=7.5$ for mode T2, approximately corresponding to maximally amplified linear conditions. In all four cases an isosurface of the magnitude of the normalized disturbance vorticity is plotted, at an arbitrarily chosen level of 0.1 , within the domain $(x \in[0,1]) \times(y \in$ $[0,1]) \times\left(z \in\left[0, L_{z}\right]\right)$, where $L_{z}=2 \pi / \beta$ indicates one (spanwise) periodicity length. Most activity takes place in the neighbourhood of the upstream wall of the cavity, $x=0$, as has already been reported by Ding \& Kawahara $(1998 a, b)$ for mode T2, 


\begin{tabular}{ccccc}
\multicolumn{5}{c}{ Mode } \\
& \multicolumn{5}{c}{$\mathrm{T} 1$} & $\mathrm{~T} 2$ & $\mathrm{~T} 3$ \\
\cline { 2 - 5 } Aspect ratio & \multicolumn{5}{c}{$\operatorname{Re}_{\text {crit }}$} \\
0.5 & 1593.94 & 1466.89 & 1630.02 & 1712.44 \\
1 & 782.61 & 844.57 & 922.10 & 960.54 \\
2 & 541.05 & 544.32 & 360.09 & 620.11 \\
3 & 541.03 & 468.60 & 303.76 & 581.17 \\
4 & 596.20 & 290.00 & 561.22 & 572.90 \\
\multicolumn{5}{c}{$\beta_{\text {crit }}$} \\
0.5 & 31.31 & 13.13 & 7.20 & 31.91 \\
1 & 15.37 & 15.77 & 7.40 & 14.31 \\
2 & 21.11 & 11.24 & 5.70 & 10.93 \\
3 & 10.91 & 8.75 & 5.00 & 11.04 \\
4 & 11.40 & 5.00 & 4.20 & 10.10 \\
& \multicolumn{5}{c}{$f_{\text {crit }} \equiv 2 \pi /\left\{\omega_{\text {r }}\right\}$ crit } \\
0.5 & 0 & 0.1663 & 0.0760 & 0.2221 \\
1 & 0 & 0.1012 & 0.0790 & 0.2145 \\
2 & 0 & 0.0905 & 0.0926 & 0.2164 \\
3 & 0 & 0.0919 & 0.0819 & 0.2202 \\
4 & 0 & 0.0901 & 0.0118 & 0.2216
\end{tabular}

TABLE 7. Critical parameters of the least-stable modes as function of aspect ratio.
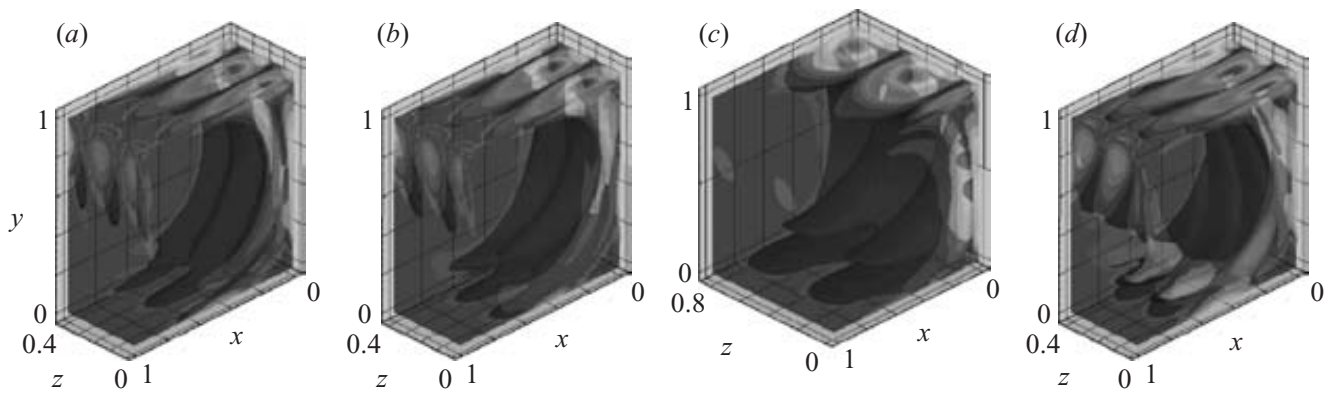

FIGURE 8. Spatial distribution of the magnitude of disturbance vorticity of the mostunstable eigenmodes in the square lid-driven cavity. (a) S1 $(R e=1000, \beta=15),(b) \mathrm{T} 1(R e=$ $1000, \beta=15),(c) \mathrm{T} 2(\operatorname{Re}=1000, \beta=7.5)$, and $(d) \mathrm{T} 3(\operatorname{Re}=1000, \beta=15)$.

with two distinct disturbance peaks within each periodicity length identifiable on the cavity lid, $y=1$, in all eigenmodes. Overall, however, there are very few qualitative differences to be found in the spatial structure of the eigenfunctions of the four most unstable cavity modes.

From the point of view of identification, either in experiment or by DNS, of the global eigenmodes documented here we note the following. S1 is the only amplified eigenmode in the region $R e \in[785,845]$; since this is a stationary mode its amplification is expected to be observed as a spanwise modulation of the steady flow, having a periodicity length related to the most unstable wavenumber by $L_{z}=2 \pi / \beta \approx 0.41$ cavity length/depth units. If the Reynolds number is taken in the range $R e \in[845,925]$ the travelling mode T1 also becomes unstable. Since the 
wavenumber vector is normal to the plane on which the steady basic flow develops, the complex conjugate of the T1 eigenmode is also a solution of the disturbance equations (3.12)-(3.15) and the two complex-conjugate modes form a standing wave pattern which grows at the rate of T1, linearly superimposed upon the growing S1 mode. On the other hand, the most amplified wavenumbers of S1 and T1 lie quite close to each other and the only qualitative difference with the situation in $R e \in[785,845]$ is the ability to measure by power-spectral analysis the frequency of the travelling mode T1. Contrary to the conjecture put forward by Ding \& Kawahara (1998b), it appears that it was mode T1 that was measured by Benson \& Aidun (1992). At $R e \in[925,960]$ the situation changes significantly on account of the amplification of mode T2. This instability has a frequency distinct from that of T1, which should also be identifiable in the spectrum (but has not been reported by Benson \& Aidun 1992). Interestingly, the most unstable wavenumber of the T2 eigenmode is approximately half that of the two previously amplified global eigenmodes, such that the linear amplification of T2 alongside that of S1 and T1 may be mistakenly perceived as amplification of a subharmonic of mode T1. This picture changes quantitatively (but not qualitatively) by the amplification of $\mathrm{T} 3$ at $R e>960$, since on the one hand an additional frequency is introduced but on the other hand the most unstable wavenumber of $\mathrm{T} 3$ is in the neighbourhood of those of $\mathrm{S} 1$ and $\mathrm{T} 1$ and the linear or nonlinear amplification of the three stronger eigenmodes will dominate the dynamics of the transition process beyond this Reynolds number value. Renewed experimental or DNS efforts focusing on the Reynolds number ranges discussed here and aided by the results of table 7 are desirable in order to identify the nonlinear modifications to the above linear laminar-turbulent transition scenario. However, unlike the cases of the rectangular duct and the wall-bounded Couette flow, flow in the lid-driven cavity appears to undergo laminar-turbulent transition on account of a linear, albeit BiGlobal, instability mechanism.

As a final note on the square LDC flow, we address the question of unsteadiness of the two-dimensional lid-driven cavity flow and the well-documented discrepancy between two-dimensional (Ghia et al. 1982; Schreiber \& Keller 1983) and threedimensional (Kim \& Moin 1985) numerical simulation results in the lid-driven cavity. We return to the square lid-driven cavity, for which it is well-established that the upper limit for the Reynolds number below which a steady-state solution can be obtained in two-dimensional simulations is $R e \approx 10^{4}$. This result is put on a firm theoretical basis using the present BiGlobal linear theory by monitoring the linear amplification of the two-dimensional $(\beta=0)$ global eigenmode. To this end we have obtained steady two-dimensional basic flows in the square cavity in $R e \in\left[10^{2}, 0.8 \times 10^{4}\right]$ and present in figure 9 the results of solution of the eigenvalue problem (3.12)(3.15) for the two-dimensional global mode. The progressive destabilization of the two-dimensional basic flow can be inferred from this result, which also explains the increasingly long integration times required to obtain a steady state when solving the time-dependent equations of motion as $R e$ increases, in terms of the diminishing magnitude of the damping rate of the least-stable global flow eigendisturbance. Regarding the discrepancy between two- and three-dimensional simulation results, it is clear that this will manifest itself at supercritical conditions for mode S1. While a two-dimensional DNS will produce steady-state solutions of the equations of motion above $R e \approx 783$, a three-dimensional simulation above this Reynolds number value, in which $L_{z}=2 \pi / \beta$ is chosen according to the present theoretical results, will initially show exponential amplification of mode S1, leading to nonlinearity and departure from the two-dimensional steady-state solution. 


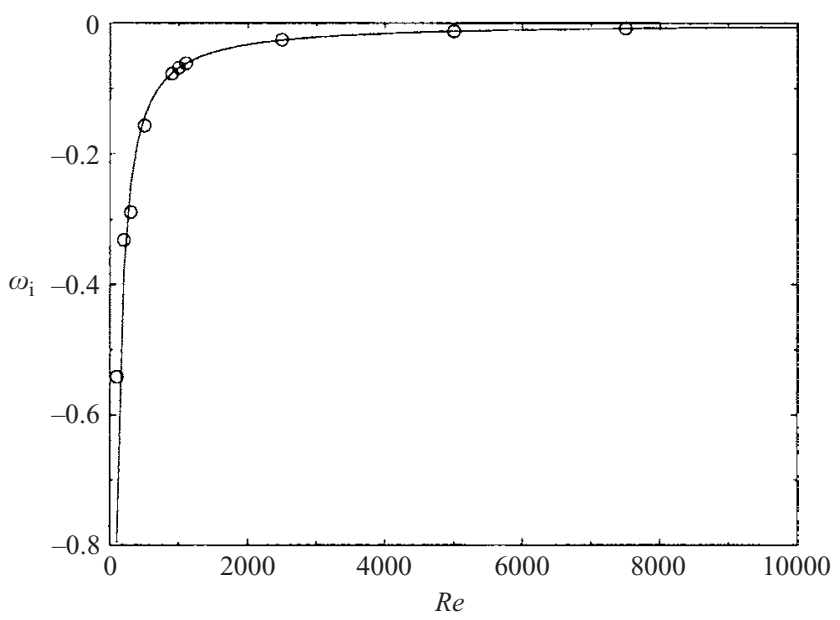

FiguRE 9. Dependence of the damping rate $\omega_{\mathrm{i}}$ of the least-damped two-dimensional $(\beta=0)$ global eigenmode on $R e$ in the square lid-driven cavity. Symbols indicate the Reynolds number values at which the partial-derivative eigenvalue problem was solved.

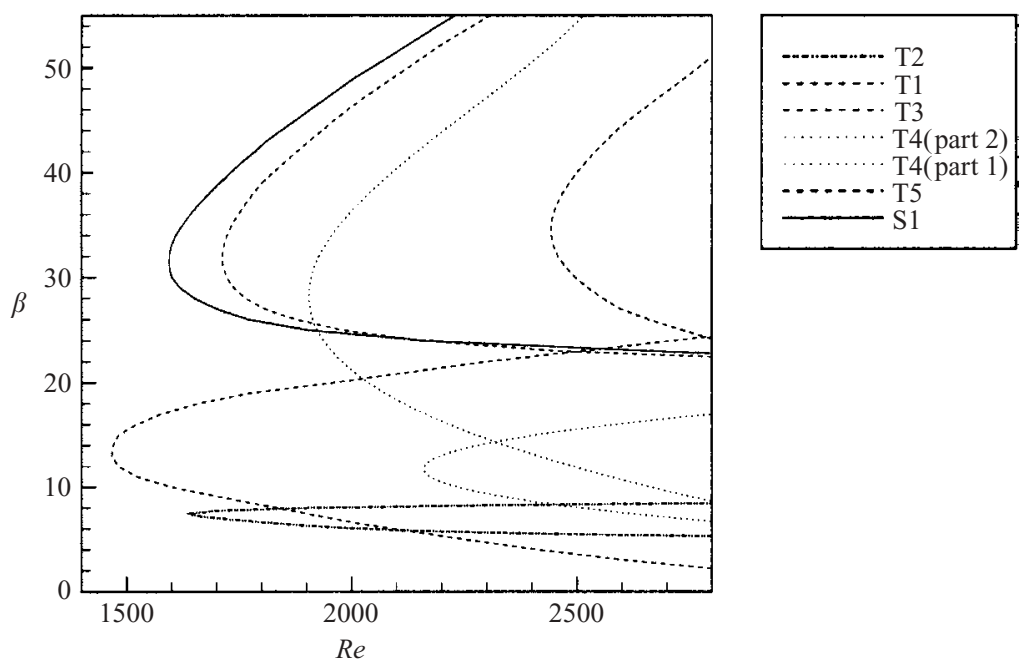

FIGURE 10. Neutral modes of a rectangular lid-driven cavity. $A=0.5$.

\subsubsection{The rectangular lid-driven cavity}

Next we investigate the effect of variable aspect ratio on the instability of the lid-driven cavity flow. Figures 10-13 show the neutral loops of the most-unstable modes in cavities of aspect ratio $A=0.5,2,3$ and 4 (see also figure 7 for unity aspect ratio results). The critical values are also tabulated in table 7 and the following observations are made. An increase of the aspect ratio is consistently found to have a destabilizing effect on all eigenmodes of the lid-driven cavity flow. At the lowest aspect ratio value examined, $A=0.5$, a multitude of modes have been found with at least three of them having critical Reynolds numbers $R e_{\text {crit }} \in[1400,1700]$. At this aspect ratio the travelling mode $\mathrm{T} 1$ is the most unstable eigendisturbance, closely 


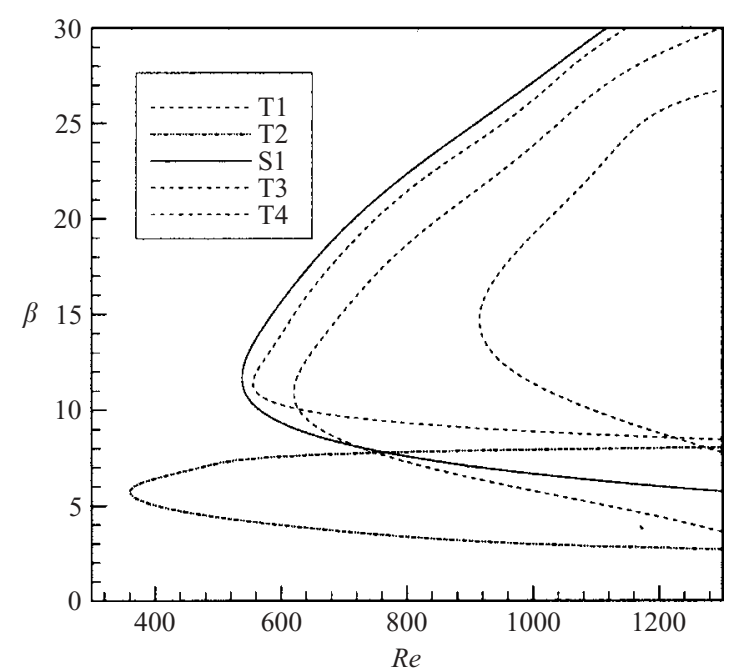

FIGURE 11. Neutral modes of a rectangular lid-driven cavity. $A=2$.

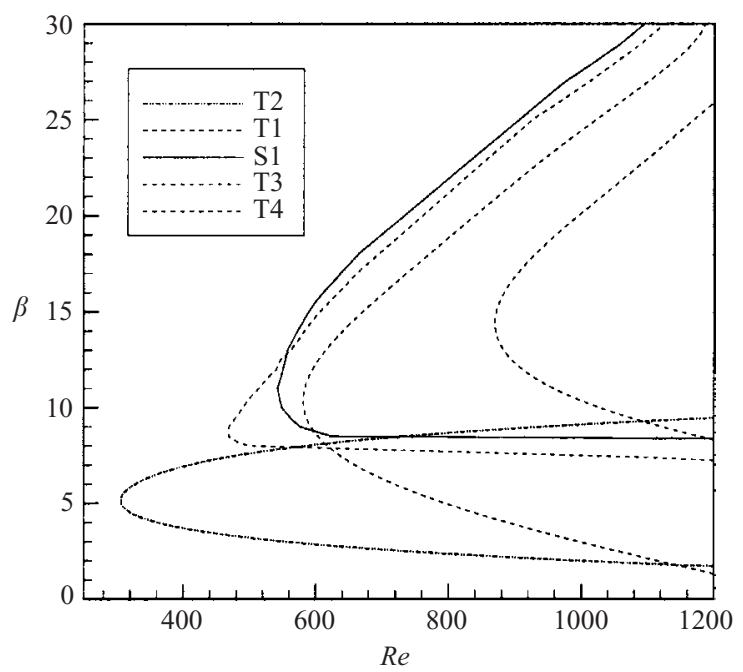

FIGURE 12. Neutral modes of a rectangular lid-driven cavity. $A=3$.

followed by the stationary mode $\mathrm{S} 1$ and the travelling mode $\mathrm{T} 2$. At $A=1$, as has been seen, $\mathrm{S} 1$ overtakes T1, which is in turn more unstable than T2 and T3. A qualitative difference between the $A=0.5$ and $A=1$ results is that by contrast to the former, in the latter case the stationary and the travelling modes T1 and T3 are clustered in approximately the same wavenumber region around $\beta=15$, while $\mathrm{T} 2$ is isolated from the rest, centred around $\beta=7.5$. The doubling of the aspect ratio from $A=0.5$ to 1 results in an approximate halving of the critical Reynolds number to $R e_{\text {crit }} \approx 800 . \dagger \mathrm{A}$ further increase of the aspect ratio from $A=1$ to $A=2$ again results in approximately

$\dagger$ The most unstable mode at the different aspect ratios does not remain the same and the concept of a critical Reynolds number is used here to signify that pertaining to the most unstable mode at each aspect ratio value 


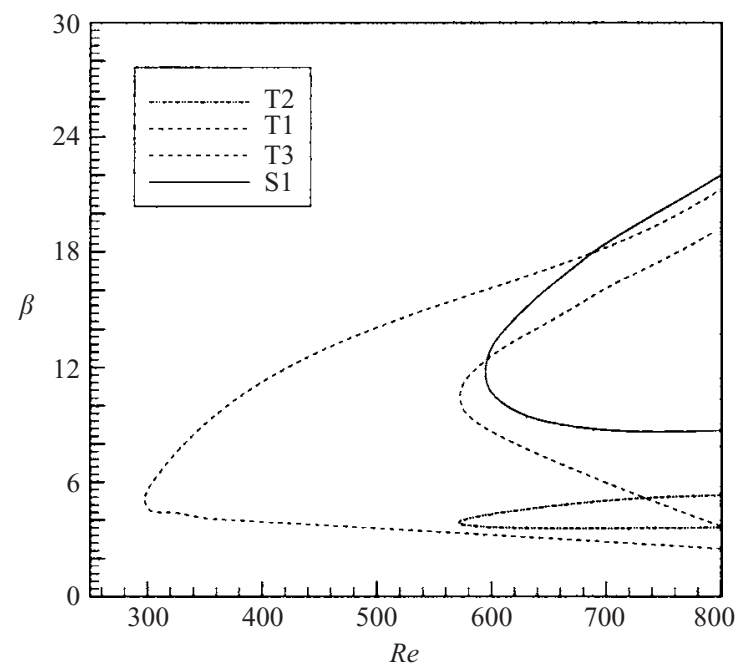

FIGURE 13. Neutral modes of a rectangular lid-driven cavity. $A=4$.

halving the critical Reynolds number value from $R e_{\text {crit }} \approx 800$ at $A=1$ to $R e_{\text {crit }} \approx 400$ at $A=2$. Indeed, it could be argued that a Reynolds number based on cavity width rather than depth might be more appropriate, but then this would be inconsistent with previous published work on cavity flows. The separation around the tips of the neutral loops between mode T2 on the one hand and all other modes on the other persists at $A=2$; one striking difference between the results at $A=1$ and 2 is that in the latter case mode T2 has overtaken all other BiGlobal instabilities in the cavity in such a way that it is the only unstable disturbance in a wide Reynolds number range $R e \in[360,580]$. The results at $A=3$ are qualitatively quite similar to those at $A=2$, with mode T2 being the most-unstable disturbance, although here mode T1 again turns out to be more unstable than mode S1. A quantitative difference between the results at $A=2$ and 3 is that the wavenumbers of the unstable modes are already merging together at about $R e=500$ for the latter aspect ratio as opposed to almost 800 for the former, such that the experimental identification of the various eigenmodes in an $A=3$ cavity may be very difficult past $R e \approx 500$. A further twist in the picture is seen in the $A=4$ results. Although modes $\mathrm{S} 1, \mathrm{~T} 2, \mathrm{~T} 3, \ldots$ are all more stable than their lower aspect ratio counterparts, the instability of the flow is again dominated by that of mode $\mathrm{T} 1$, which encompasses all wavenumber ranges of all other unstable eigenmodes. Mode $\mathrm{T} 1$ at $A=4$ has a critical Reynolds number $R e_{\text {crit }} \approx 300$, approximately half that of the other modes at this aspect ratio value. The results on the dependence of the critical Reynolds number on the aspect ratio are summarized in figure 14 for the four most-unstable eigenmodes. It may be seen that a sharp decrease of the critical Reynolds numbers of the different modes as the aspect ratio increases from low values is followed by much milder variation of $R e_{\text {crit }}$ beyond $A \geqslant 2$ where the critical Reynolds numbers of modes $\mathrm{S} 1$ and $\mathrm{T} 3$ approximately remain constant at $R e \approx 600$, while that of mode $\mathrm{T} 1$ reaches a minimum at $A \approx 2.75$ followed by an increase with $A$, up to the highest aspect ratio values examined. The critical Reynolds number of mode $\mathrm{T} 1$ decreases with increasing aspect ratio, but then reaches a minimum at approximately the $A$ value where $R e_{\text {crit }}$ of T2 has a minimum, also. Two crossover points between most unstable disturbances may be singled out on this plot, $(A, R e) \approx(1.2,720)$ and $(3.5,400)$; the first corresponds to the location in parameter 


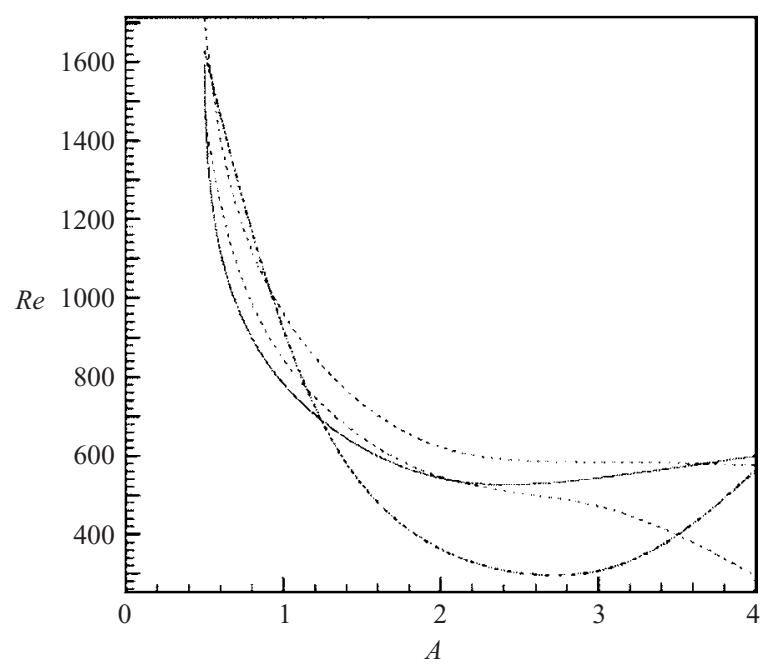

FIGURE 14. The critical Reynolds number of the most-unstable lid-driven cavity modes as a function of the cavity aspect ratio. Line styles as figure 12 .

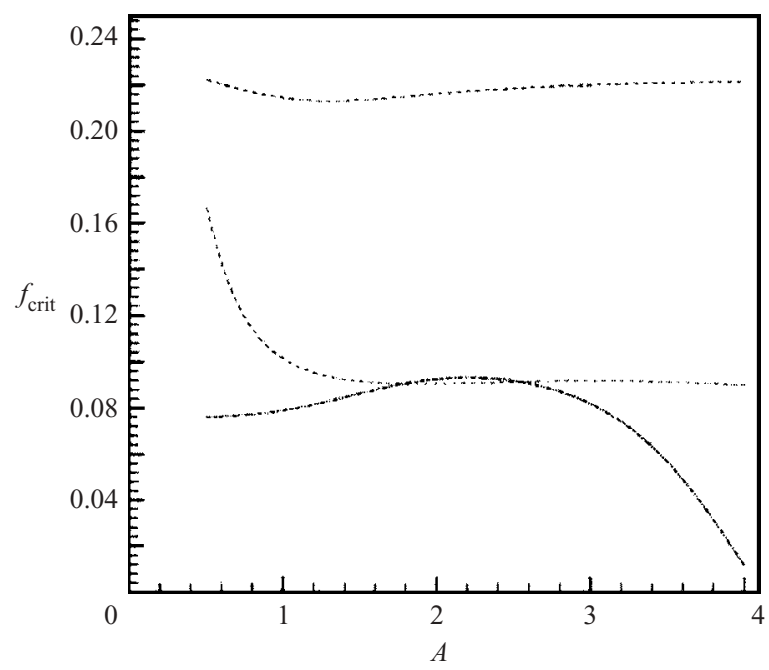

FIGURE 15 . The critical frequency of the most-unstable lid-driven cavity modes as a function of the cavity aspect ratio. Line styles as figure 12 .

space where the most-unstable eigenmode of the cavity changes from a stationary to a pair of travelling eigenmodes, while at the second point the most unstable eigenmode changes its character from a T2 to T1 disturbance. A crossover between the T1 and T2 modes is also to be observed at $A<1$, where its significance is moderated by the fact that at the latter conditions mode $\mathrm{S} 1$ is the most unstable eigendisturbance. This interpretation of the dependence of critical Reynolds number on aspect ratio is intricately complicated by the results of figure 15 , where the critical frequency of the same modes is plotted against aspect ratio. It is seen that the frequency of eigenmode $\mathrm{T} 3$ is well separated from that of modes T1 and T2 for all but the lowest aspect ratio value examined and remains practically constant with aspect ratio. The constancy of the frequency of $\mathrm{T} 2$ with aspect ratio at $A>1$ is also to be seen in these results. 
However, eigenmode T1 appears to have a frequency close to that of T3 at low aspect ratio values which smoothly merges into that of $\mathrm{T} 2$ at $A$ slightly above unity up to about $A=3$; then, the frequency of mode T2 sharply decreases with $A$. The conclusion from the latter observation is that the only discriminating characteristic to discern experimentally between eigenmodes $\mathrm{T} 2$ and $\mathrm{T} 3$ at $A>1$ is the respective critical Reynolds numbers and most unstable wavenumbers. However, the closeness of the frequencies may make isolated observation of the two modes at $A>1$ rather difficult. The present theoretical results suggest that experimental identification of these distinct eigenmodes should be undertaken at $A \leqslant 1$. In this respect, we note that the domain in which the basic flow is obtained (and the subsequent instability analysis is performed) has an influence on the BiGlobal eigenmode characteristics. In their recent work, Albensoeder et al. (2001b) have examined the stability of rectangular liddriven cavity flow. However, we were unable to confirm the results of these authors in spite of a number of very careful computations (and taking into account the different non-dimensionalizations when studying rectangular geometries), and in spite of the quite good agreement in the square geometry case.

\subsection{The $2 D C-L D C$ flow}

We finally turn our attention to the flow established in the duct shown in figure 1 by motion of the lid at a constant speed of unity and angles $\phi \in(0, \pi / 2)$. In view of the requirements posed by the subsequent stability analysis on the quality of the underlying basic flows, as identified in the preceding LDC flow stability analysis, $128^{2}$ spectral collocation points have been used for the recovery of the 2DC-LDC basic states analysed in this section. All steady states have been obtained at a single aspectratio value of $A=1$, at three angle values $\phi=\pi / 8, \pi / 4$ and $3 \pi / 8$ and several Reynolds number values, $R e \in[0,1000]$, of which only those interesting from a stability analysis point of view, $R e=800,900$ and 1000 will be discussed in what follows. Numerical solutions to the partial-derivative eigenvalue problem in this class of flows require a level of computational effort which is an order-of-magnitude higher than that in the LDC flow class. The reason is the existence of all three basic flow velocity components in the 2DC-LDC flow which results in the need to solve a complex two-dimensional eigenvalue problem. The computing effort is then comparable with that in the pressure-gradient-driven duct and the wall-bounded Couette flows, and can be met by present-day hardware (Theofilis et al. 2003).

At $R e=800$ and all three values of $\phi$ examined, a scan of the wavenumber range $\beta \in[0,25]$ did not deliver unstable eigenmodes. On the other hand, several such modes were found at the higher Reynolds number values studied, $R e=900$ and 1000 ; results are summarized in figure 16 where unstable eigenmodes obtained at three angles $\phi=\pi / 8, \pi / 4$ and $3 \pi / 8$ and $\beta \in[0,25]$ are shown.

At $\phi=\pi / 8$ one observes the counterparts of modes T1, T2, T3 and T4 of the LDC flow, a result which points to the continuity and persistence of these modes in the 2DC-LDC flow at small values of $\phi$. At $\phi=\pi / 8$ relatively short wavelengths are amplified, the range of $L_{z}=2 \pi / \beta$ extending approximately in the range $L_{z} \in$ $(0.3,1.25)$. This range corresponds to that of maximally amplified instabilities in the $\phi=0$ (LDC) flow. As the angle $\phi$ increases, several interesting effects can be observed. First, all modes are damped substantially; the maximum amplification rate at $\phi=\pi / 4$ is a factor two smaller than that at $\phi=\pi / 8$. Second, at $\phi=\pi / 4$ modes amalgamate together so that at this angle it is difficult to identify the discrete LDC modes found at $\phi=0$ and their counterparts found in the 2DC-LDC flow at $\phi=\pi / 8$. In an intriguing, and probably counter-intuitive manner in view of the results of $\S 4.2$, a further increase 
(a)
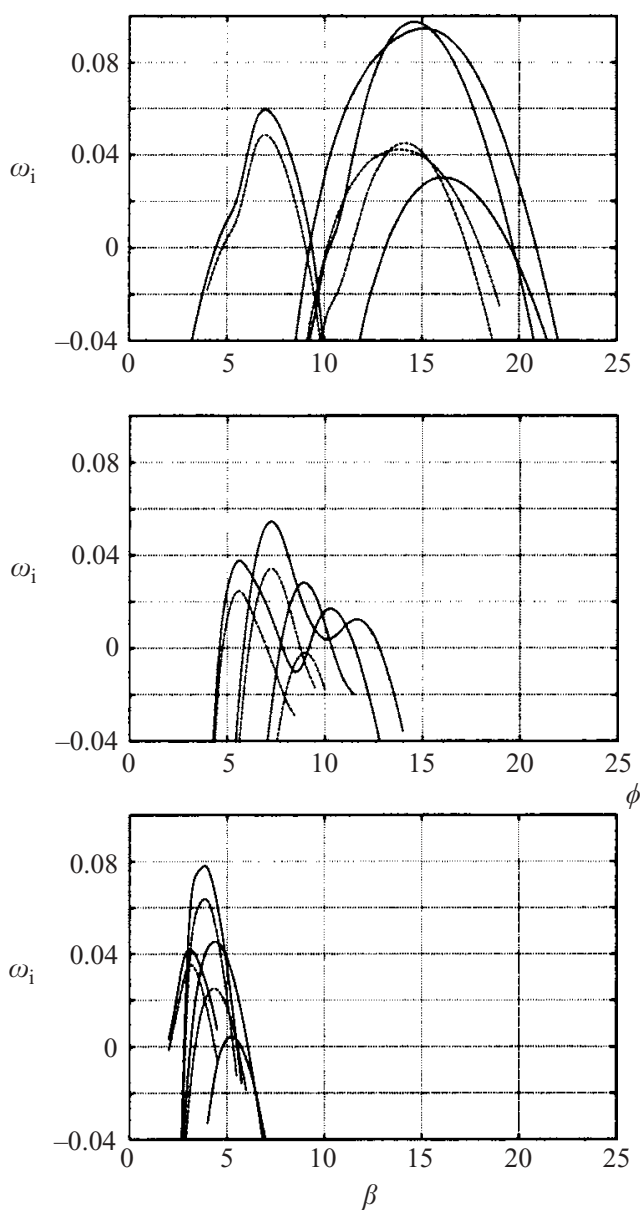

$\phi=\pi / 8$

(b)

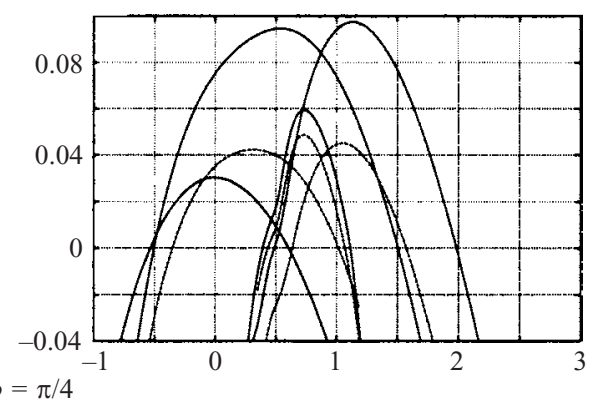

$\phi=\pi / 4$

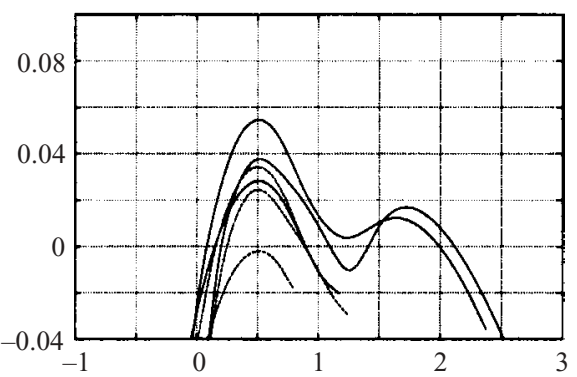

$\phi=3 \pi / 8$

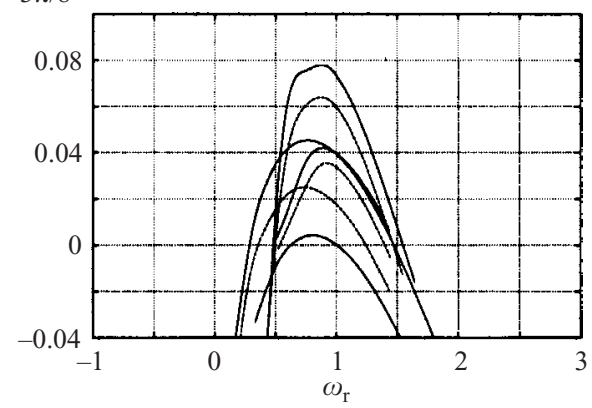

FIGURE 16. Dependence of $\omega_{\mathrm{i}}$ on $\beta(a)$ and $\omega_{\mathrm{r}}(b)$ at three angles $\phi=\pi / 8, \pi / 4$ and $3 \pi / 8$ and two Reynolds numbers $R e=900(\cdots)$ ) and $1000(-$ ).

of the angle to $\phi=3 \pi / 8$ results in an increase of the amplification rates compared to those at $\phi=\pi / 4$ back to levels corresponding to those at $\phi=\pi / 8$. The range of amplified wavenumbers systematically shrinks as $\phi$ is increased; consequently, the periodicity lengths within which amplified disturbances may be found change from (approximately) the values of LDC flow at $\phi=\pi / 8$ to $L_{z} \in(0.5,1.25)$ at $\phi=\pi / 4$ and then shift to $L_{z} \in(1,3)$ at $\phi=3 \pi / 8$.

Turning to the dependence of the amplification rates on the disturbance frequencies, also shown in figure 16, one notes that an increase of $\phi$ results in a systematic decrease of the range of amplified $\omega_{\mathrm{r}}$. Another interesting aspect of the frequencies of the amplified eigendisturbances identified in the present results is the near-linear dependence of $\omega_{\mathrm{r}}$ of the amplified modes on $\beta$, seen in figure 17. Table 8 quantifies this effect in terms of slopes of the straight lines $\omega_{\mathrm{r}}=\omega_{\mathrm{r}}^{*}+\lambda\left(\beta-\beta^{*}\right)$ approximating the dependence of $\omega_{\mathrm{r}}$ on $\beta$ of the different modes examined. Finally, in all results obtained the (expected) stabilizing effect of lowering the Reynolds number at constant $\phi$ may be observed. However, it should be noted that this effect is different for the different modes and is substantially more pronounced at low $\phi$ values. 

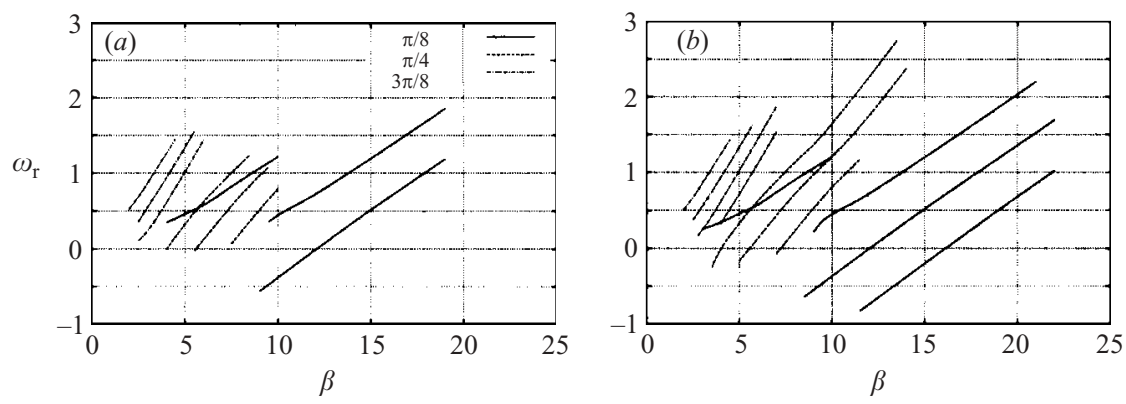

FIGURE 17. Dependence of $\omega_{\mathrm{r}}$ on $\beta$ at three angles $\phi=\pi / 8, \pi / 4$ and $3 \pi / 8$ and two Reynolds numbers $(a) R e=900$ and $(b) 1000$.

$\begin{array}{ccccc}\phi & \lambda & \left(\beta^{*}, \omega_{\mathrm{r}}^{*}\right)_{1} & \left(\beta^{*}, \omega_{\mathrm{r}}^{*}\right)_{2} & \left(\beta^{*}, \omega_{\mathrm{r}}^{*}\right)_{3} \\ \pi / 8 & 1 / 6 & (8,1.0) & (15,1.2) & (15,0.5) \\ \pi / 4 & 0.3 & (10,0.75) & (7,0.5) & (6,0.5) \\ 3 \pi / 8 & 0.4 & (5,1.0) & (4,1.0) & (3,1.0)\end{array}$

TABLE 8. Slopes $\lambda$ and coordinates $\left(\beta^{*}, \omega_{r}^{*}\right)$ of the points defining the straight lines which approximate the linear dependence of the frequency $\omega_{\mathrm{r}}$ of three amplified eigenmodes on the spanwise wavenumber $\beta$ in 2DC-LDC flow at $R e=900$ and three $\phi$ values.

\section{Discussion}

Our concern here has been with three-dimensional global linear instability of four classes of two-dimensional steady basic flows developing in rectangular enclosures. Flow has been taken to be homogeneous along the axial direction of the ducts considered, permitting an expansion of eigendisturbances in normal modes along that spatial direction. The other two spatial directions are fully resolved, leading to a two-dimensional, partial-derivative eigenvalue problem describing temporal linear stability of the respective flows. This global (BiGlobal) eigenvalue problem has been solved numerically, using spectral collocation and Krylov subspace iteration for the recovery of the leading eigenvalues.

In the first flow addressed, pressure-gradient-driven flow in a rectangular duct defined by four stationary walls, we have fully confirmed the linear instability results of Tatsumi \& Yoshimura (1990) who used a somewhat different numerical procedure to that of the present work. No new modes were found in the rectangular duct, despite extensive numerical experimentation using an approach in which attention is not confined to modes of special symmetries. The effect of the introduction of lateral walls in a linear framework is not sufficient to explain the discrepancies between experimental (Kao \& Park 1970) and theoretical (Tatsumi \& Yoshimura 1990) results. On the contrary, lateral walls were found by Tatsumi \& Yoshimura (1990) to stabilize the PPF eigenspectrum; here, lateral walls were shown to introduce additional $\left(\omega_{\mathrm{r}}=\right.$ const, $\left.\omega_{\mathrm{i}} \rightarrow-\infty\right)$ branches analogous to the P-family of PPF, (Mack 1976), all of which have $\omega_{\mathrm{r}}<2 / 3$, while in the limit of a square duct the S-family of PPF is found to split in two parts of stable disturbances. In the second flow examined here, lateral walls are introduced to form the wall-bounded analogue of the PCF, in which flow is driven by the constant-speed motion of one wall along the homogeneous direction; in this direction the pressure gradient is taken to be 
zero. Only stable eigenmodes were found in wall-bounded Couette flow. In a manner analogous to PCF, its eigenspectrum was found to be confined in $\omega_{\mathrm{r}} \in[-1,1]$ and also to have the familiar Y-shape.

By contrast to these two flows, in which the basic flow direction and the wavenumber vector are parallel, in the third flow considered, the classic lid-driven cavity, the wavenumber vector is normal to the plane in which the basic flow varies. Here, linear instability analysis has delivered linearly unstable modes in very good agreement with experiment. We found that the inconsistencies in previous theoretical results can be attributed to insufficient resolution in two of the three past analyses, namely those predicting the lowest and the highest critical Reynolds number values. Very good agreement is obtained between the results of Ding \& Kawahara (1998b) and our calculations, as far as the mode quoted in the past as delivering the critical conditions is concerned. However, in line with the predictions of Theofilis (2000) and Albensoeder et al. (2001b), we find that this mode is the third in significance from an instability analysis point of view. The frequency of this travelling mode T2 shows only qualitative agreement with that measured experimentally, the discrepancy being of $O(80 \%)$ in the range of Reynolds numbers monitored experimentally. In a parameter range unexplored by earlier investigations we have found two new modes which are more strongly amplified than the known mode T2. The first mode S1 is stationary and becomes unstable at $R e_{\text {crit }} \approx 780$; this mode may only be perceived as a modification of the two-dimensional steady state. The critical Reynolds number of the second mode $\mathrm{T} 1, \operatorname{Re} \approx 840$, places it well inside the experimentally available bracket; its frequency is within $1 \%$ of that identified experimentally. Several modes amplified supercritically to $\mathrm{T} 2$ were also found to exist in the lid-driven cavity flow.

We have also considered the effect that a variable cavity aspect ratio has on the instability of the flow, in the regime $A \in[0.5,4]$. Outside this domain global instability analysis is extremely challenging on account of either the high critical Reynolds number values encountered at low aspect ratios or the large surface area to be resolved at large aspect ratios; at both extremes the resolution requirements are inaccessible by currently available hardware and algorithms. With this in mind, we have found an increase in the aspect ratio of the lid-driven cavity to have a destabilizing effect on all of the most unstable modes; the critical Reynolds number $R e_{\text {crit }}$ of the most unstable global eigenmode at each aspect ratio varies from $R e_{\text {crit }}>1.5 \times 10^{3}$ as $A \rightarrow 0$ to $R e_{\text {crit }} \approx 300$ at the highest aspect ratio value examined, $A=4$. An additional result of interest from a flow-control point of view is that the frequency of the most-unstable modes were found to be linear functions of and very weakly dependent on Reynolds number past the tips of the respective neutral loops.

Finally, we have investigated the origin of the paradox of erroneous linear theory predictions in the wall-bounded Couette flow class and very good agreement of linear theory and experiment in the lid-driven cavity class of flows. Basic flows set up by lid-motion at an angle $\phi \in(0, \pi / 2)$, possessing all three velocity components, were analysed with respect to their linear instability. All flows examined become linearly unstable above $R e=800$. At small angles $\phi$ and analogous $R e$ ranges all four unstable travelling LDC modes were found to be present in the 2DC-LDC flow also. By contrast, no evidence of the (most unstable) stationary mode S1 of LDC flow was found in the 2DC-LDC flow. As $\phi$ increased a decrease of the amplification rates with respect to the LDC and small- $\phi 2$ DC-LDC flows was observed at $\phi=\pi / 4$, followed by recovery of the amplification rates when $\phi$ is increased further. This result leads to the conjecture that basic flow motion on the (resolved) $(x, y)$-plane is necessary for the appearance of linearly unstable modes in lid-driven cavity flows. 
In summary, the systematic numerical experimentation in the present paper has on the one hand left unanswered the question of subcritical instability in the rectangular duct and has added one more erroneous prediction of linear stability of wall-bounded Couette flow. On the other hand, linear analysis has proved successful in predicting instability in line with experimental findings in the classic lid-driven cavity and has delivered unstable modes in the combined wall-bounded Couette and lid-driven cavity flow.

This work was sponsored in part by the Air Force Office of Scientific Research, USAF, under grant number FA8655-03-1-3059 to Nu Modelling, S. L., C/. Mariano Barbacid 1/9, E-28660 Boadilla del Monte (Madrid), Spain. The grant is monitored by Mr Wayne Donaldson (EOARD) and Dr J. Schmisseur (AFOSR). The views and conclusions contained herein are those of the authors and should not be interpreted as necessarily representing the official policies or endorsements, either expressed or implied, of the Air Force Office of Scientific Research or the US Government. Additional support for this work was provided by a Ramón y Cajal research fellowship of the Spanish Ministry of Science and Technology (V.T.) and the Engineering and Physical Sciences Research Council (J.O.).

\section{REFERENCES}

Aidun, C. K., Triantafillopoulos, N. G. \& Benson, J. D. 1991 Global stability of a lid-driven cavity with throughflow: Flow visualization studies. Phys. Fluids A 3, 2081-2091.

Albensoeder, S., Kuhlmann, H. C. \& Rath, H. J. $2001 a$ Multiplicity of steady two-dimensional flows in two-sided lid-driven cavities. Theor. Comput. Fluid. Dyn. 14, 223-241.

Albensoeder, S., Kuhlmann, H. C. \& Rath, H. J. $2001 b$ Three-dimensional centrifugal-flow instabilities in the lid-driven-cavity problem. Phys. Fluids 13, 121-135.

Barkley, D., Gomes, M. G. M. \& Henderson, R. D. 2002 Three-dimensional instability in flow over a backward facing step. J. Fluid Mech. 473, 167-190.

BARKLEY, D. \& Henderson, R. 1996 Three-dimensional floquet stability analysis of the wake of a circular cylinder. J. Fluid Mech. 322, 215-241.

Benson, J. D. \& Aidun, C. K. 1992 Transition to unsteady nonperiodic state in a through-flow lid-driven cavity. Phys. Fluids A 4, 2316-2319.

Boberg, L. \& Brosa, U. 1988 Onset of turbulence in a pipe. Z. Naturforsch. 43a, 697-726.

Bottin, S., Dauchot, O., Daviaud, F. \& Manneville, P. 1998 Experimental evidence of streamwise vortices as finite amplitude solutions in transitional plane Couette flow. Phys. Fluids 10, 2597-2607.

Bruneau, C. \& Jouron, C. 1990 An efficient scheme for solving the incompressible Navier-Stokes equations. J. Comput. Phys. 89, 389.

Burggraf, O. R. 1966 Analytical and numerical studies of the structure of steady separated flows. J. Fluid Mech. 24, 113-151.

Clever, R. \& Busse, F. 1997 Tertiary and quarternary solutions in plane Couette flow. J. Fluid Mech. 344, 137-153.

Daviaud, F., Hegseth, J. \& Bergé, P. 1992 Subcritical transition to turbulence in plane Couette flow. Phys. Rev. Lett. 69, 2511-2514.

Deville, M., Fischer, P. \& Mund, E. 2002 High-order Methods for Incompressible Fluid Flow. Cambridge University Press.

Ding, Y. \& Kawahara, M. 1998a Linear stability of incompressible flow in a cavity using finite element method. Intl J. Numer. Meth. Fluids 27, 139-157.

Ding, Y. \& Kawahara, M. $1998 b$ Linear stability of incompressible flow using a mixed finite element method. J. Comput. Phys. 139, 243-273.

Ding, Y. \& KAWAHARA, M. 1999 Three-dimension linear stability analysis of incompressible viscous flows using the finite element method. Intl J. Numer. Meth. Fluids 31, 451-479. 
Dongarra, J., Straughan, B. \& Walker, D. 1996 Chebyshev tau - QZ algorithm methods for calculating spectra of hydrodynamic stability problems. J. Appl. Numer. Maths 22, 399-435.

Drazin, P. \& ReID, W. 1981 Hydrodynamic Stability. Cambridge University Press.

Eckhardt, B., Marzinzik, K. \& Schmiegel, A. 1998 Transition to turbulence in shear flows. In A Perspective Look at Nonlinear Media (ed. J. Parisi, S. Müller \& W. Zimmermann), pp. 327-338. Springer.

Ehrenstein, U. 1996 On the linear stability of channel flows over riblets. Phys. Fluids 8, 3194-3196.

FAisst, H. \& ECKhardt, B. 2000 Transition from the Couette-Taylor to the plane Couette system. Phys. Rev. E 61, 7227-7230.

Freitas, C., Street, R., Findikakis, A. \& Koseff, J. R. 1985 Numerical simulations of three dimensional flow in a cavity. Intl J. Numer. Meth. Fluids 5, 561-575.

Ghia, U., GHia, K. \& Shin, C. 1982 High-Re solutions for incompressible flow using the NavierStokes equations and a multigrid method. J. Comput. Phys. 48, 387-411.

Goodrich, J. W., Gustafson, K. \& Halasi, K. 1990 Hopf bifuraction in the driven cavity. J. Comput. Phys. 90, 219-261.

Guj, G. \& Stella, F. 1993 A vorticity-velocity method for the numerical solution of 3-d incompressible flows. J. Comput. Phys. 106, 286-298.

Gupta, M. 1991 High accuracy solutions of incompressible Navier-Stokes equations. J. Comput. Phys. 93, 343.

Hall, P. \& Horseman, N. 1991 The linear inviscid secondary instability of longitudinal vortex structures in boundary layers. J. Fluid Mech. 232, 357-375.

HärTel, C. J. \& MeibuRG, E. 1999 Gravity currents and their analysis by direct numerical simulation. Tech. Rep. Reihe 7, Nr. 366. VDI.

Henningson, D. 1987 Stability of parallel inviscid shear flow with mean spanwise variation. Tech. Rep. FFA Report TN 1987-57. FFA.

Herbert, T. 1974 Über endliche Amplituden periodischer Störungen der Grenzschicht an der ebenen Platte. Tech. Rep. FB 74-53. DLR, engl. Transl. ESA TT-169 (1975).

Herbert, T. 1977 Finite amplitude stability of plane parallel flows. In AGARD CP-224, pp. 3-1-3-10.

HockInG, L. M. 1977 The stability of flow in an elliptic pipe with large aspect ratio. Q. J. Mech. Appl. Maths 30, 343-353.

Hocking, L. M. 1978 Nonlinear instability of flow in a rectangular pipe with large aspect ratio. J. Appl. Maths Phys. 29, 100-111.

Huerre, P. \& Monkewitz, P. 1990 Local and global instabilities in spatially developing flows. Annu. Rev. Fluid Mech. 22, 473-537.

JaCkson, A. P., Hillier, R. \& Soltani, S. 2001 Experimental and computational study of laminar cavity flows at hypersonic speeds. J. Fluid Mech. 427, 329-358.

Jacquin, L., Fabre, D., Sipp, D., Theofilis, V. \& Vollmers, H. 2003 Instability and unsteadiness of aircraft trailing vortices. Aerospace Sci. Tech. 7, 577-593.

KaO, T. \& PARK, C. 1970 Experimental investigations of the stability of channel flows. Part 1. flow of a single liquid in a rectangular channel. J. Fluid Mech. 43, 145-164.

Karniadakis, G. E. \& Sherwin, S. J. 1999 Spectral/hp element Methods for CFD. Oxford University Press.

Kerswell, R. \& Davey, A. 1996 On the linear instability of elliptic pipe flow. J. Fluid Mech. 316, $307-324$.

KIM, J. \& MoIN, P. 1985 Application of a fractional-step method to incompressible Navier-Stokes equations. J. Comput. Phys. 59, 308-323.

KLEISER, L. 1982 Numerische Simulationen zum Laminar-Turbulenten Umschlagprozess der ebenen Poiseuille-Strömung. PhD thesis, Inst. Reaktorenentwicklung KFZ Karlsruhe.

Koseff, J. \& Street, R. 1984 The lid-driven cavity flow: A synthesis of qualitative and quantitative observations. Trans. ASME: J. Fluids Engng 106, 390-398.

Ku, H. C., Hirsh, R. S. \& Taylor, T. D. 1987 A pseudospectral method for solution of the three-dimensional Navier-Stokes equations. J. Comput. Phys. 70, 439-462.

LeE, N. Y., Schultz, W., W. \& Boyd, J. P. 1989 Stability of fluid in a rectangular enclosure by spectral method. Intl J. Heat Mass Transfer 32, 513-520.

Lin, R.-S. \& MALIK, M. R. 1996 On the stability of attachment-line boundary layers. Part 1. the incompressible swept hiemenz flow. J. Fluid Mech. 311, 239-255. 
MACK, L. 1976 A numerical study of the temporal eigenvalue spectrum of the blasius boundary layer. J. Fluid Mech. 73, 497-520.

Moffatt, H. K. 1964 Viscous and resistive eddies near a sharp corner. J. Fluid Mech. 18, 1-18.

Morzynski, M. \& Thiele, F. 1991 Numerical stability analysis of flow about a cylinder. Z. Angew. Math. Mech. 71, T424-T428.

NAGATA, M. 1990 Three-dimensional finite-amplitude solutions in plane Couette flow: bifurcation from infinity. J. Fluid Mech. 217, 519-527.

Napolitano, M. \& Catalano, L. 1991 A multigrid solver for the vorticity-velocity Navier-Stokes equations. Intl J. Numer. Meth. Fluids 13, 49.

Nishida, H. \& SATOFuKa, N. 1992 Higher-order solutions of square driven cavity using a variableorder multigrid method. Intl J. Numer. Meth. Engng 34, 637.

Nishioka, M. \& AsaI, M. 1985 Some observations of the subcritical transition in plane Poiseuille flow. J. Fluid Mech. 150, 441-450.

NishioKa, M., IIDA, S. \& ICHIKAWA, Y. 1975 An experimental investigation of the stability of plane poiseuille flow. J. Fluid Mech. 72, 731-751.

OrszaG, S. 1971 Accurate solution of the Orr-Sommerfeld stability equation. J. Fluid Mech. 50, 689-703.

Orszag, S. \& Patera, A. 1983 Secondary instability of wall-bounded shear flows. J. Fluid Mech. 128, 347-385.

Otto, S. \& Denier, J. P. 1999 Numerical solution of a generalized elliptic partial differential eigenvalue problem. J. Comput. Phys. 156, 352-359.

PAN, F. \& ACrivos, A. 1967 Steady flows in rectangular cavities. J. Fluid Mech. 28, 643-655.

Pierrehumbert, R. T. 1986 A universal shortwave instability of two-dimensional eddies in an inviscid fluid. Phys. Rev. Lett. 57, 2157-2159.

Poliashenko, M. \& Aidun, C. K. 1995 A direct method for computation of simple bifurcations. $J$. Comput. Phys. 121, 246-260.

Ramanan, N. \& Homsy, G. M. 1994 Linear stability of lid-driven cavity flow. Phys. Fluids 6, 2690-2701.

RAYLEIGH, LORD 1914 Further remarks on the stability of viscous fluid motion. Phil. Mag. 28 (6), 609-619.

Reddy, S., Schmid, P., Baggett, J. \& Henningson, D. 1998 On the stability of streamwise streaks and transition thresholds in plane channel flows. J. Fluid Mech. 365, 269-303.

REYNOLDS, O. 1883 An experimental investigation of the circumstances which determine whether the motion of water shall be direct or sinuous, and of the law of resistance in parallel channels. Phil. Trans. R. Soc. Lond. 174, 935-982.

Romanov, V. A. 1973 Stability of plane parallel Couette flow. Funkcional Anal. i Proložen 7 (2), $62-73$.

Rosenhead, L. 1963 Laminar Boundary Layers. Oxford University Press.

Rowley, C. W., Colonius, T. \& Basu, A. J. 2002 On self-sustained oscillations in two-dimensional compressible flow over rectangular cavities. J. Fluid Mech. 455, 315-346.

SAAD, Y. 1980 Variations of arnoldi's method for computing eigenelements of large unsymmetric matrices. Lin. Algebra Appl. 34, 269-295.

Schmid, P. \& Henningson, D. 2001 Stability and Transition in Shear Flows. Springer.

Schreiber, R. \& Keller, H. 1983 Driven cavity flows by efficient numerical techniques. J. Comput. Phys. 49, 310-333.

Schubauer, G. B. \& Skramstad, H. K. 1947 Laminar boundary layer oscillations and stability of laminar flow. J. Aero. Sci. 14, 69-78.

Seifert, A., Theofilis, V., Joslin, R. D. \& Collis, S. C. 2004 Issues in active flow control: Theory, simulation, experiment and control. Prog. Aerospace Sci. (to appear).

ShaY, W. 1981 Development of a second order approximation for the Navier-Stokes equations. Computers Fluids 9, 279.

SHEN, J. 1991 Hopf bifurcation of the unsteady regularized driven cavity flow. J. Comput. Phys. 95, 228.

Spalart, P., Moser, R. \& Rogers, M. 1991 Spectral methods for the Navier-Stokes equations with one infinite and two periodic directions. J. Comput. Phys. 96, 297-324.

Stocker, J. \& Duck, P. 1995 Stationary perturbations of Couette-Poiseuille flow: the flow development in long cavities and channels. J. Fluid Mech. 292, 153-182. 
TAng, L., Cheng, T. \& Tsang, T. 1995 Transient solutions for three-dimensional lid-driven cavity flows by a least-squares finite-element method. Intl J. Numer. Meth. Fluids 21, 413-432.

TAtsumi, T. \& Yoshimura, T. 1990 Stability of the laminar flow in a rectangular duct. J. Fluid Mech. 212, 437-449.

TheofiLis, V. 2000 Globaly unstable basic flows in open cavities. AIAA Paper 00-1965.

TheofiLis, V. 2003 Advances in global instability of nonparallel and three-dimensional flows. Prog. Aerospace Sci. 39 (4), 249-315.

Theofilis, V., Fedorov, A., Obrist, D. \& Dallmann, U. C. 2003 The extended Görtler-Hämmerlin model for linear instability of three-dimensional incompressible swept attachment-line boundary layer flow. J. Fluid Mech. 487, 271-313.

Thompson, M. \& Ferziger, J. 1989 An adaptive mutigrid technique for the incompressible NavierStokes equations. J. Comput. Phys. 82, 94.

Tillmark, N. \& Alfredsson, P. H. 1992 Experiments on transition in plane Couette flow. J. Fluid Mech. 235, 89-102.

Tollmien, W. 1929 Über die Entstehung der Turbulenz. Nach. Ges. Wiss. Göttingen, pp. 21-44.

Trefethen, L., Trefethen, A. E., Reddy, S. C. \& Driscoll, T. 1993 Hydrodynamic stability without eigenvalues. Science 261, 578-584.

Tuann, S.-Y. \& Olson, M. 1978 Review of computing methods for recirculating flows. J. Comput. Phys. 29, 1.

Tumin, A. 2000 Onset of turbulence in circular pipe flows. In Proc. IUTAM Laminar-Turbulent Symposium V (ed. W. Saric \& H. Fasel), pp. 373-382. Sedona, AZ, USA.

WALEFFE, F. 1995 Transition in shear flows. Nonlinear normality versus non-normal linearity. Phys. Fluids 7, 3060-3066.

Wilkinson, J. H. 1965 The Algebraic Eigenvalue Problem. Clarendon.

Wintergerste, T. \& Kleiser, L. 2000 Secondary stability analysis of nonlinear crossflow vortices. In Proc. IUTAM Laminar-Turbulent Symposium V (ed. W. Saric \& H. Fasel), pp. 583-586. Sedona, AZ, USA.

Zang, T. \& Hussaini, M. 1995 Numerical experiments on subcritical transition mechanism. AIAA Paper 85-0296. 